\title{
Granulation in K-type dwarf stars
}

\section{Hydrodynamic simulations and 3D spectrum synthesis}

\author{
I. Ramírez ${ }^{1,2}$, C. Allende Prieto ${ }^{2,3}$, L. Koesterke ${ }^{4}$, D. L. Lambert ${ }^{2}$, and M. Asplund ${ }^{1}$ \\ 1 Max Planck Institute for Astrophysics, Postfach 1317, 85741 Garching, Germany \\ e-mail: ivan@mpa-garching.mpg.de \\ 2 McDonald Observatory and Department of Astronomy, University of Texas, Austin, TX 78712-0259, USA \\ 3 Mullard Space Science Laboratory, University College London, Holmbury St. Mary, Dorking, Surrey RH5 6NT, UK \\ 4 Texas Advanced Computing Center, University of Texas, J. J. Pickle Research Campus, Austin, TX 78758-4497, USA
}

Received 28 January 2009 / Accepted 7 May 2009

\begin{abstract}
Aims. To explore the impact of surface inhomogeneities on stellar spectra, granulation models need to be computed. Ideally, the most fundamental characteristics of these models should be carefully tested before applying them to the study of more practical matters, such as the derivation of photospheric abundances. Our goal is to analyze the particular case of a K-dwarf.

Methods. We construct a three-dimensional radiative-hydrodynamic model atmosphere of parameters $T_{\text {eff }}=4820 \mathrm{~K}, \log g=4.5$, and solar chemical composition. Using this model and 3D spectrum synthesis, we computed a number of Fe I and Fe II line profiles. The observations presented in the first paper of this series were used to test the model predictions. The effects of stellar rotation and instrumental imperfections are carefully taken into account in the synthesis of spectral lines.

Results. The theoretical line profiles show the typical signatures of granulation: the lines are asymmetric, with their bisectors having a characteristic $\mathrm{C}$-shape and their core wavelengths shifted with respect to their laboratory values. The line bisectors span from about 10 to $250 \mathrm{~m} \mathrm{~s}^{-1}$, depending on line strength, with the stronger features showing larger span. The corresponding core wavelength shifts range from about $-200 \mathrm{~m} \mathrm{~s}^{-1}$ for the weak Fe I lines to almost $+100 \mathrm{~m} \mathrm{~s}^{-1}$ in the strong Fe I features. Based on observational results for the Sun, we argue that there should be no core wavelength shift for Fe I lines of $E W \gtrsim 100 \mathrm{~m} \AA$. The cores of the strongest lines show contributions from the uncertain top layers of the model, where non-LTE effects and the presence of the chromosphere, which are important in real stars, are not accounted for. The Fe II lines suffer from stronger granulation effects due to their deeper formation depth which makes them experience stronger temperature and velocity contrasts. For example, the core wavelength shifts of the weakest Fe II lines are about $-600 \mathrm{~m} \mathrm{~s}^{-1}$. The comparison of model predictions to observed Fe I line bisectors and core wavelength shifts for our reference star, HIP 86400, shows excellent agreement, with the exception of the core wavelength shifts of the strongest features, for which we suspect inaccurate theoretical values. Since this limitation does not affect the predicted line equivalent widths significantly, we consider our 3D model validated for photospheric abundance work.
\end{abstract}

Key words. stars: atmospheres - stars: late-type - sun: granulation

\section{Introduction}

Solar granulation is the visible manifestation of surface convection (e.g., Bray et al. 1984; Muller 1999). When rising gas from the convective zone reaches the photosphere, it cools down by radiation losses. Since the opacity decreases steeply with decreasing gas temperature, the temperature of the photosphere drops abruptly as hydrogen recombines and the gas becomes suddenly transparent. The upflowing material in the granules decelerates as it enters the convectively stable region due to the higher pressures above and flows sideways until encountering other granules before being accelerated downwards due to it now being overdense and having negative buoyancy. Since convective envelopes are present in other cool stars (spectral type F and later on the main sequence, $\mathrm{G}$ and later for giants and supergiants), it is expected that they also experience surface granulation.

The physics of radiatively driven convection is complex; it is three-dimensional, time-dependent, non-local, and non-linear. Parameterized models of granulation may require a large number of free parameters whose physical interpretation is obscure. Furthermore, they do not guarantee a unique solution. On the other hand, the continuous growth of computer power is allowing us to solve this problem numerically using only the basic laws of hydrodynamics, including radiative transfer in the energy equation. A better understanding of solar and stellar granulation is thus possible through numerical simulations (e.g., Nordlund \& Dravins 1990; Freytag et al. 1996; Stein \& Nordlund 1998; Asplund et al. 1999; Ludwig et al. 1999; Robinson et al. 2003; Vögler 2004; Asplund 2005; Collet et al. 2006, 2007; Trampedach 2007; Nordlund et al. 2008).

Theoretical models can be computed at will, but using them is meaningless until we are reassured that they include the relevant physics behind the phenomenon they intend to represent. The validation of theoretical models, i.e., verifying that their most fundamental predictions are supported by targeted observations, is necessary before exploring the impact of their effects on other matters, such as the inferred chemical compositions in the case of stellar atmospheres.

Stellar disks are usually unresolved. The exceptions are nearby red supergiants, for which interferometric observations with present-day technology are in principle capable of revealing 
surface structures related to convection (e.g., Gilliland \& Dupree 1996; Chiavassa 2008). In any case, there are no near-future prospects for direct observations of stellar granulation.

Fortunately, granulation signatures are also present in stellar spectra: net line wavelength shifts and asymmetries. Very careful observations are required to detect the effects of granulation in this manner (Dravins 1987b; Ramírez et al. 2008, hereafter Paper I). High quality data have been used by several authors to show that stars with convective envelopes experience granulation with different degrees of intensity and velocity contrasts (e.g., Gray 1982, 2005; Dravins 1987a, 2008; Allende Prieto et al. 2002). Other velocity fields may become prominent in more evolved stars (Gray et al. 2008). Indeed, Gray \& Nagel (1989) have defined a granulation boundary on the HR diagram based on observations of spectral line asymmetries in hot and cool stars. They find that the shape and magnitude of the line asymmetries in hot stars (A-type and hotter on the main sequence, F-type and hotter for the supergiants) are qualitatively different from those that correspond to solar granulation and argue that they cannot be related to deep envelope convection.

Spectrum synthesis using classical, hydrostatic model atmospheres predicts symmetric lines whose core wavelengths correspond exactly to the input laboratory wavelengths. In real stellar atmospheres that experience granulation, line profiles coming from bright, hot granules are blueshifted because the material is rising and therefore approaching the observer while line profiles coming from the dark, cool intergranular lanes are redshifted. The continuum level of the upflow line-profiles is higher than that of the line-profiles associated with the cool downflows. Thus, if the granulation pattern is unresolved, an observed line profile is dominated by the blueshifted component. The profile is then asymmetric and its observed core wavelength is blueshifted (e.g., Dravins et al. 1981). The line asymmetry can be quantified by the line bisector, defined as the location of the midpoints of horizontal segments joining the blue and red wings of the lines, and the core wavelength shifts due to granulation are often referred to as convective blueshifts.

The goal of this series of papers is to understand the surface inhomogeneities (granulation) present in K-dwarf stars and explore their impact on the determination of fundamental parameters and chemical compositions derived from the star's spectral energy distribution. In the first part of the series (Ramírez et al. 2008, hereafter Paper I), we showed that, with very careful observing strategies, it is possible to not only detect but also accurately quantify the effects of granulation on line profiles in the spectra of a few bright K-type dwarf stars. A theoretical granulation model has been calculated and it is described in detail in this paper, along with the tests made to ensure that its most fundamental predictions, namely their impact on the shapes of absorption line profiles (specifically those due to $\mathrm{Fe}$ I), are valid. This validation will allow us to explore with confidence the socalled "3D effects" on the determination of chemical compositions and fundamental parameters of K-dwarfs in the final part of this series (Ramírez et al. 2009, hereafter Paper III).

\section{The 3D model}

Assuming local thermodynamic equilibrium (LTE), we computed a three-dimensional radiative-hydrodynamic model atmosphere using the methods described in Stein \& Nordlund (1998). A plane-parallel box of the stellar envelope was modeled by solving the fluid dynamics equations of mass continuity, momentum conservation, and energy conservation (e.g., Eqs. (1)-(3) in Stein \& Nordlund 1998). The effect of temperature enters through the equation of state and by the radiative heating rate, which is determined by solving the radiative transfer equation (e.g., Eq. (7) in Stein \& Nordlund 1998).

In the calculation of the 3D model, the equation of state (as given by Mihalas et al. 1988), continuum opacities, and source functions were obtained by interpolation in the tables included in the updated MARCS stellar atmosphere package (Gustafsson et al. 1975, 2008). The adopted line opacities (opacity distribution functions) are those by Kurucz (1993a,b).

Surface gravity, chemical composition, and entropy of the gas entering the simulation box are the fundamental parameters of the $3 \mathrm{D}$ model calculation. The effective temperature is not an input parameter as in 1D models but it is rather adjusted by modifying the state of the gas entering the bottom of the computational domain until it reaches an average similar to the target value of $4820 \mathrm{~K}$. The other input parameters are $\log g=4.5$ and $[\mathrm{Fe} / \mathrm{H}]=0.0$. The solar abundances adopted are those given by Grevesse \& Sauval (1998).

The model consists of $150 \times 150 \times 82$ grid points representing a rectangular box of the following geometrical dimensions: $4.7 \times 4.7 \times 3.2 \mathrm{Mm}\left(1 \mathrm{Mm} \equiv 10^{6} \mathrm{~m}\right)$. The simulation box has periodic horizontal boundaries, gas is allowed to escape at the top, while the density and energy of the incoming gas at the bottom boundary are adjusted to conserve the entropy. After relaxation, the simulation was run for about one hour of stellar time, from which 100 snapshots separated by $40 \mathrm{~s}$ were extracted and employed for the line formation calculations presented in this study (the actual hydrodynamical time-step was approximately $0.2 \mathrm{~s}$ ).

The time evolution of the emergent intensity that would be observed on the surface of this model star is illustrated in Fig. 1 (in Sect. 3 we provide details on the calculation of the emergent intensity from the 3D model). The pattern of granules and intergranular lanes resembles that of the Sun but on a smaller geometrical scale. The correlation between the emergent intensity of a given snapshot and those of the subsequent ones decreases smoothly and vanishes for images separated by about $10 \mathrm{~min}$, a number comparable to that obtained for the solar simulation. Thus, the lifetime of granules in our K-dwarf simulation is similar to that of the Sun.

Figure 2 shows the temperature structure of our model in the upper $1 \mathrm{Mm}$ layers (this is the portion that we use for the spectral line calculations, as explained in Sect. 3). The rapid decrease of temperature with atmospheric height just below the visible surface $(\text { depth }=0)^{1}$, attributed to the feedback between radiation losses and decreasing opacity, is the most prominent feature. There, across a layer of only about $50 \mathrm{~km}$, the gas temperature drops about $3000 \mathrm{~K}$. The apparent discontinuity of the temperature structure at depth $\simeq 0.1 \mathrm{Mm}$ is due to this effect. In the regions of line formation (depth $<0$ ), the temperature fluctuations have extremes that differ by about $1000 \mathrm{~K}$.

Also shown in Fig. 2 are one-dimensional models computed with the ATLAS (Kurucz 1979, 1993a) and MARCS (Gustafsson et al. 1975) codes $^{2}$. It is clear that, in the regions of line-formation, 1D and 3D models show very similar temperature structures if, for the latter, the average value of the

\footnotetext{
1 The "depth" variable corresponds to the geometrical depth, increasing inward, and it is zero at the plane where the spatially-averaged Rosseland optical depth along the vertical direction is equal to one.

2 The references given here correspond to those that describe the basic properties of the models. Several improvements have been made since their publication. The models presented in Fig. 2 are those available at: http: //kurucz . harvard. edu/grids .html (the "odfnew" version) and http://marcs.astro.uu.se/ (see also Gustafsson et al. 2008, for more details on the updated MARCS models).
} 

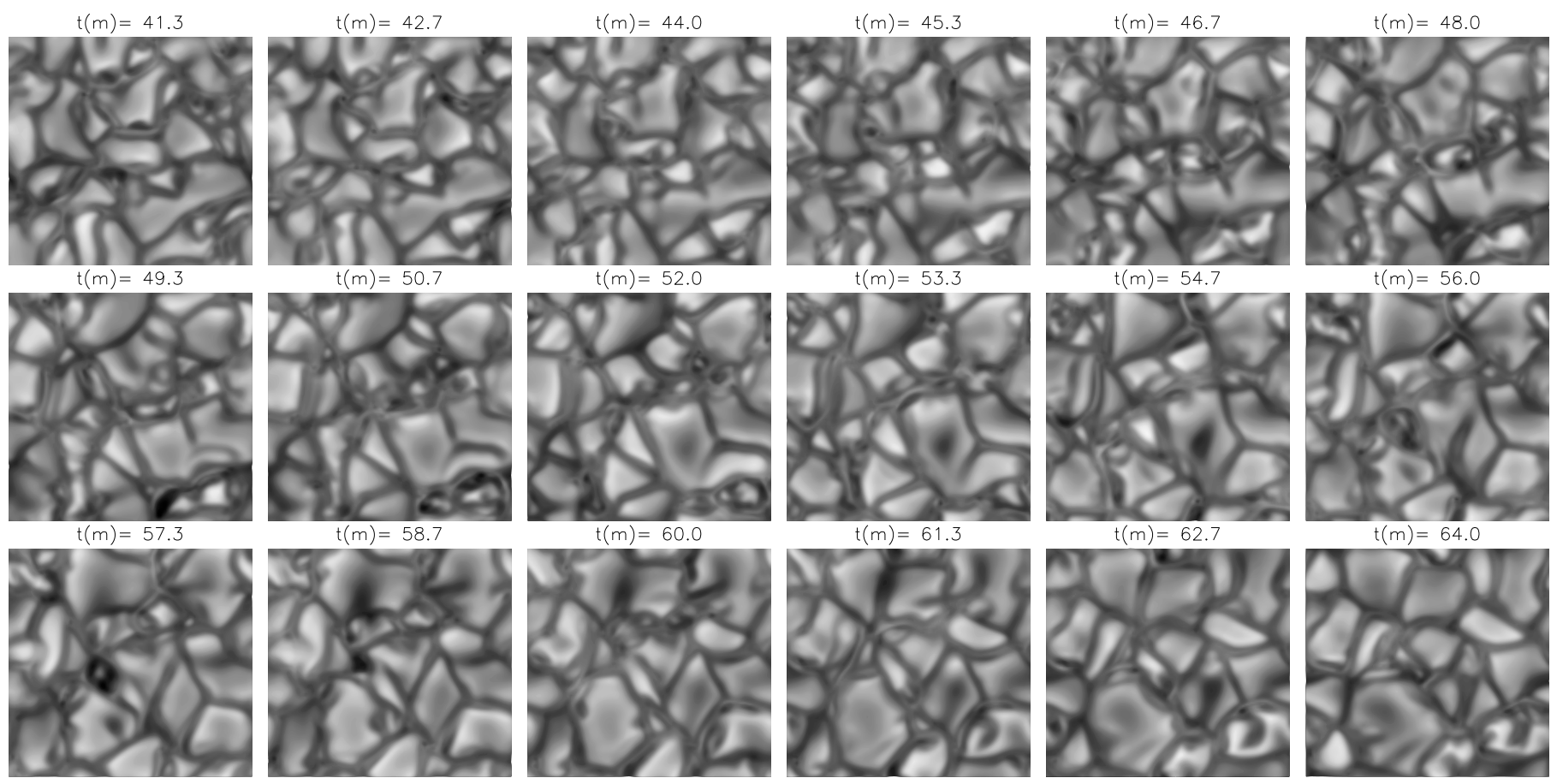

Intensity $\left(10^{-5} \mathrm{erg} \mathrm{s}^{-1} \mathrm{~cm}^{-2} \mathrm{sr}^{-1} \mathrm{~Hz}^{-1}\right)$

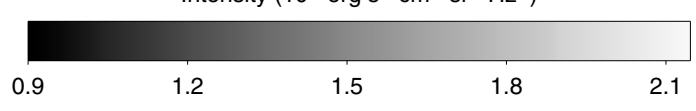

Fig. 1. Snapshots of the emergent intensity (continuum) at $6151 \AA$ predicted by our 3D K-dwarf model atmosphere (details on the spectrum synthesis are given in Sect. 3). Each panel shows an area of $4.7 \times 4.7 \mathrm{Mm}^{2}$ which corresponds to the dimensions of a horizontal cross-section of the simulation box. The time, in minutes, is given on top of each panel. Only the last 22.7 min of the simulation are shown.

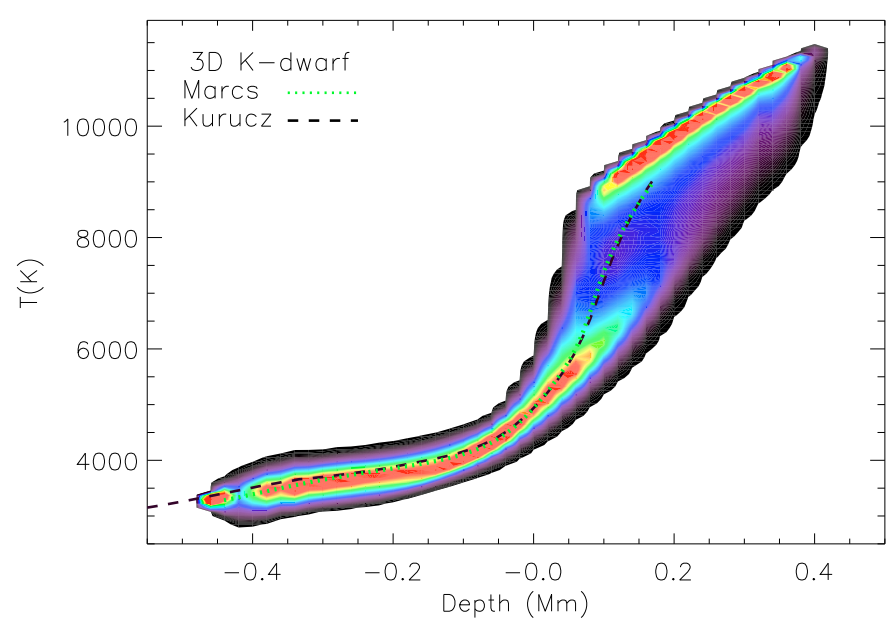

Fig. 2. Temperature structure of our 3D K-dwarf model atmosphere near the visible surface (the full simulation extends much deeper than shown here). The color code represents the number of grid points at a given temperature and depth, with red being the largest and dark blue the lowest number. The dotted green line and dashed black line correspond to temperature structures of 1D model atmospheres (see legend on the top left). The depth scale is set to zero at the geometrical plane where the mean Rosseland optical depth is equal to 1; i.e., at the "visible" surface.

temperature is obtained at each depth. We must warn, however, that it is because of the temperature (and velocity) fluctuations that the 3D model is more realistic and that this "similarity" with 1D models should not be used to argue that 1D and 3D models of K-dwarf photospheres are equivalent. The reason why the average $3 \mathrm{D}$ and $1 \mathrm{D}$ temperature structures are similar is that
3D models are nearly in radiative equilibrium at solar metallicity (e.g., Stein \& Nordlund 1998), which is not the case for metal-poor stars (e.g., Asplund et al. 1999).

A snapshot of the temperature structure of our 3D model is shown in Fig. 3. Clearly, the strongest temperature contrasts occur well below the visible surface. This phenomenon was referred to as "hidden" granulation by Nordlund \& Dravins (1990) in their study of a granulation model for $\alpha$ Cen B (spectral type $\mathrm{K} 1 \mathrm{~V}$ ) but note that the intensity fluctuations are still clearly visible on the surface of this cool star model, as illustrated by our Fig. 1.

The temperature and velocity fields near the geometrical surface of zero depth are shown in Fig. 4 for a given snapshot of the simulation (more precisely, this corresponds to a layer located $50 \mathrm{~km}$ below the visible surface). Hot granules have the largest upward velocities and appear to be expanding from their center. The horizontal velocity field converges toward intergranular lanes, which are cooler and have the largest downward velocities. This correlation between the vertical velocity and temperature fields is illustrated also in Fig. 5. Note that the correlation is tighter at a given optical depth than geometrical depth. Similar results are predicted for the density and vertical velocity fields; the downflows contain higher density gas. Thus, the predicted granulation of the 3D K-dwarf model has many characteristics of the well-observed solar granulation.

The fluctuations of the temperature, velocity, and density fields in the 3D simulation as a function of depth are quantified by their root mean square (rms) values. The maximum rms values of all these parameters occur at a depth of about $+0.1 \mathrm{Mm}$ (Fig. 6). In this layer, the rms values are about $1200 \mathrm{~K}, 2 \mathrm{~km} \mathrm{~s}^{-1}$ (vertical component only), and $0.8 \times 10^{-7} \mathrm{~g} \mathrm{~cm}^{-3}$. In the regions 


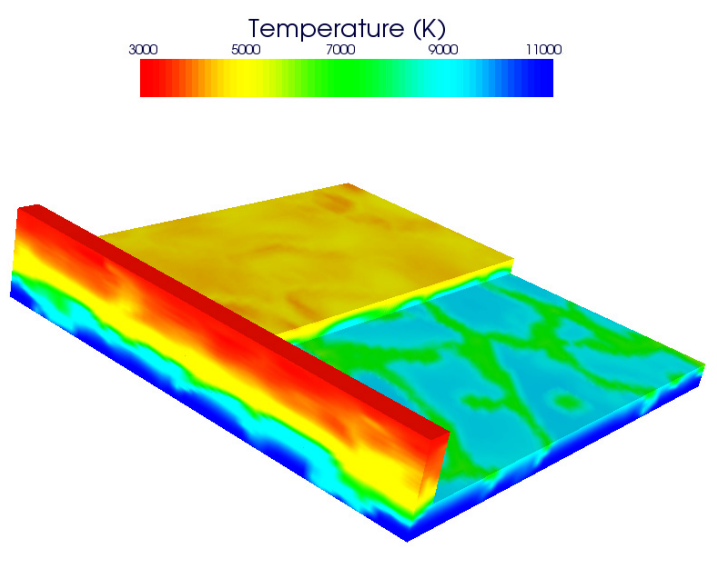

Fig. 3. Temperature structure of a snapshot of our 3D K-dwarf model atmosphere near the visible surface $($ depth $=0)$. The cross-section on the front wall shows the overall behavior of the granulation temperature field, which becomes cooler in the higher layers but is inhomogeneous at any given depth. Two horizontal cuts are shown; one at depth $=-0.15 \mathrm{Mm}$ (predominant colors are green and blue) and another one at depth $=0$ (predominant color is yellow). The granulation pattern is clearly seen at depth $=-0.15 \mathrm{Mm}$. At the visible surface, the inhomogeneities persist but to a lesser extent and the granulation pattern is much weaker.

of line formation (depth $<0$ ), the rms temperature is roughly constant at about $200 \mathrm{~K}$ while the rms vertical velocity decreases with height, from about $1.3 \mathrm{~km} \mathrm{~s}^{-1}$ at the visible surface to $0.5 \mathrm{~km} \mathrm{~s}^{-1}$ near the top of the simulation box.

Similar 3D simulations for the Sun (Stein \& Nordlund 1998; Asplund et al. 2000b, $T_{\text {eff }}=5777 \mathrm{~K}, \log g=4.44,[\mathrm{Fe} / \mathrm{H}]=0$ ) and Procyon (Allende Prieto et al. 2002, $T_{\text {eff }}=6500 \mathrm{~K}, \log g=$ $4.0,[\mathrm{Fe} / \mathrm{H}]=-0.05)$ are available for comparison. The temperature and velocity contrasts are stronger in the Procyon than in the solar model. At the visible surface $($ depth $=0)$, for example, the rms temperature in Procyon is about $8 \%$ compared to $4.5 \%$ in the solar case. In the K-dwarf model, the corresponding value is even lower, about $4.0 \%$. The peak rms velocities of the Procyon, Sun, and K-dwarf model are 5, 4, and $2 \mathrm{~km} \mathrm{~s}^{-1}$, respectively. Thus, there is clearly an effective temperature dependence such that hotter stars experience stronger temperature and velocity contrasts. This fact has been explained by Nordlund \& Dravins (1990). Basically, the convective flux scales with the total flux so that cooler stars transport less convective energy than hotter stars. In addition, cooler stars are denser and therefore lower velocities are enough to transport the convective flux. Note also that Procyon has a lower surface gravity, which makes its photosphere less dense, thus enhancing the effect.

The granulation pattern is driven by the sharp decline in the gas temperature due to radiation losses and the subsequent lowering of the extremely temperature dependent continuous opacity. In the Procyon model, this occurs very close to the visible surface whereas it occurs a few tens to hundreds of $\mathrm{km}$ below it in the cooler models (about $100 \mathrm{~km}$ in our K-dwarf case; see Fig. 2). Thus, in addition to having stronger temperature and velocity contrasts, hotter 3D models such as for Procyon have their granulation pattern visible on their surfaces, a phenomenon labeled "naked granulation" by Nordlund \& Dravins (1990).

Although in the cooler models, such as our K-dwarf simulation, the strongest temperature contrasts occur below the visible surface, the velocity fields associated with them reach larger heights, in particular the regions of line formation. For example, Fig. 6 clearly shows that that rms velocity does not vanish up to

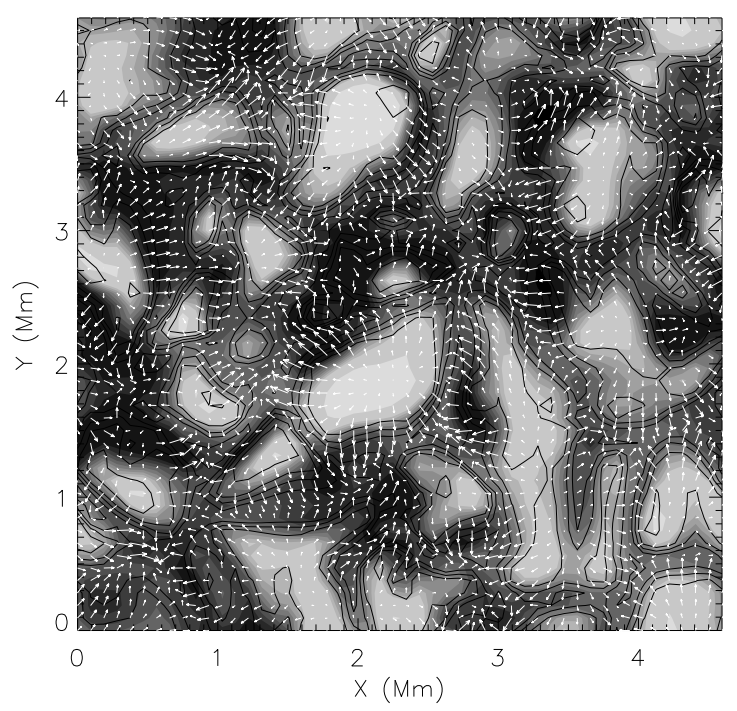

Fig. 4. Temperature and velocity fields of a snapshot of our 3D K-dwarf model atmosphere at the geometrical depth corresponding to $50 \mathrm{~km}$ below the visible surface. The velocity field is superimposed on the temperature field and is represented with solid lines (contours tracking the vertical component) and arrows (horizontal component). In this snapshot, the temperature extremes differ by about $2500 \mathrm{~K}$ (cf. Fig. 2) while the vertical velocities range from about -4 to $+4 \mathrm{~km} \mathrm{~s}^{-1}$ and the horizontal component from 0 to $5.6 \mathrm{~km} \mathrm{~s}^{-1}$ with a mean value of $2.1 \mathrm{~km} \mathrm{~s}^{-1}$.

$400 \mathrm{~km}$ above the visible surface. We therefore expect to see the granulation effects on absorption lines in K-dwarf spectra.

\section{Spectrum synthesis in 3D}

Single spectral lines were computed with the 3D synthesis code "Ite" (e.g., Asplund et al. 2000b), which is described below. In Appendix A, we compare some of these calculations with those obtained using a different 3D spectrum synthesis program, Ass $\epsilon \mathrm{t}$ (Koesterke et al. 2008), which we will use in the third part of this series (Ramírez et al. 2009) for the synthesis of continua, molecular features, and wide spectral regions. As we show in Appendix A, the agreement between the results obtained with the two codes is excellent.

The transfer equation was solved along several rays throughout the upper $0.8 \mathrm{Mm}$ of the simulation box. The contribution from deeper layers to the emergent flux is negligible, thus justifying the use of only the upper layers in the line calculations. To improve the vertical sampling, which is necessary due to the rapid change of the local physical parameters in this region and the sensitivity of the line opacities to these quantities, the upper $0.8 \mathrm{Mm}$ layers of the simulation box were interpolated to a grid of 82 depth points. In the horizontal direction, of the original $150 \times 150$ grid, a coarser $50 \times 50$ grid was adopted by eliminating two of every three grid points along the $x$ and $y$ axes, thus keeping the same geometrical extent. This has the advantage of significantly reducing computing time while keeping the results essentially unchanged (Asplund et al. 2000a). The model used for the line calculations is therefore a $50 \times 50 \times 82$ grid.

The time interval between the snapshots of the $3 \mathrm{D}$ model that were saved from the original simulation is about $40 \mathrm{~s}$. For the spectral line calculations, we used only every other saved snapshot, as tests showed that using all of them produced essentially the same results. The maximum difference in flux from 

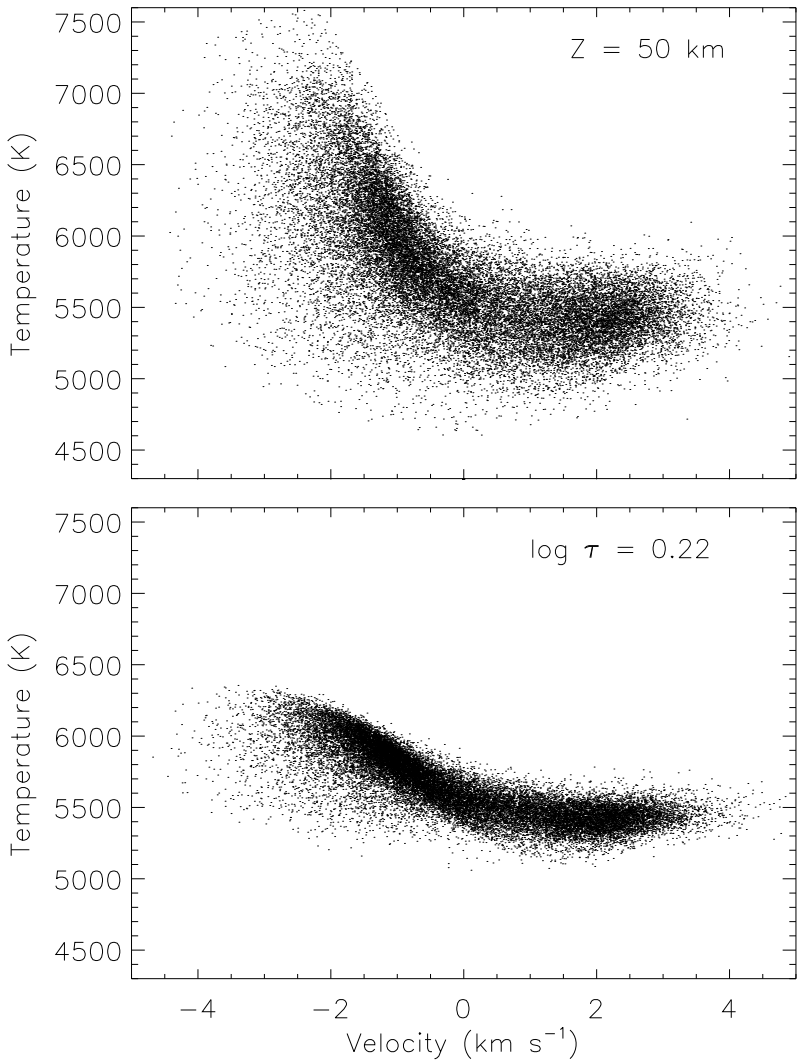

Fig. 5. Top panel: correlation between temperature and vertical velocity of the 3D K-dwarf model atmosphere at the geometrical depth corresponding to $50 \mathrm{~km}$ below the visible surface, where the average optical depth is $\langle\log \tau\rangle=0.22$. Bottom panel: as in the top panel but for local optical depth $\log \tau=0.22$.

this experiment was from less than $0.01 \%$ for weak lines to about $0.04 \%$ for the strongest lines. Tests using one every four and one every eight saved snapshots showed that this maximum flux difference increased by factors of only two (i.e., about $0.08 \%)$ and three $(0.12 \%)$, respectively. In addition to taking into account the time dependence of the granulation phenomenon, the use of several snapshots can be interpreted as the equivalent of increased spatial coverage.

We solved the equation of radiative transfer using 8 polar ( $\mu=\cos \theta$ in the standard stellar atmosphere notation) and $8 \mathrm{az}-$ imuthal $(\phi)$ angles. The number of frequencies adopted in each case was 71 , with a fine frequency spacing corresponding to $0.4 \mathrm{~km} \mathrm{~s}^{-1}$. For strong lines, this frequency sampling was not enough to reach the full extent of the wings and we therefore repeated the calculation using 71 frequencies with a spacing of $1.5 \mathrm{~km} \mathrm{~s}^{-1}$. We did this instead of calculating the whole wide spectral range with fine spacing to save computing time. The two results were then merged into single line profiles.

Similarly to the model atmosphere computation, LTE level populations and ionization fractions were adopted in the line calculations. Look-up tables for the equation of state and opacity for the wavelength region in which the problem spectral line is located were calculated before solving the radiative transfer. Interpolation from these tables during the spectral line synthesis instead of on-the-fly calculations reduced dramatically the computing time.

Each spectral line was computed for 3 different values of the oscillator strength $(\log g f$ and $\log g f \pm 0.5)$. Note that this is equivalent to computing each spectral line for three different

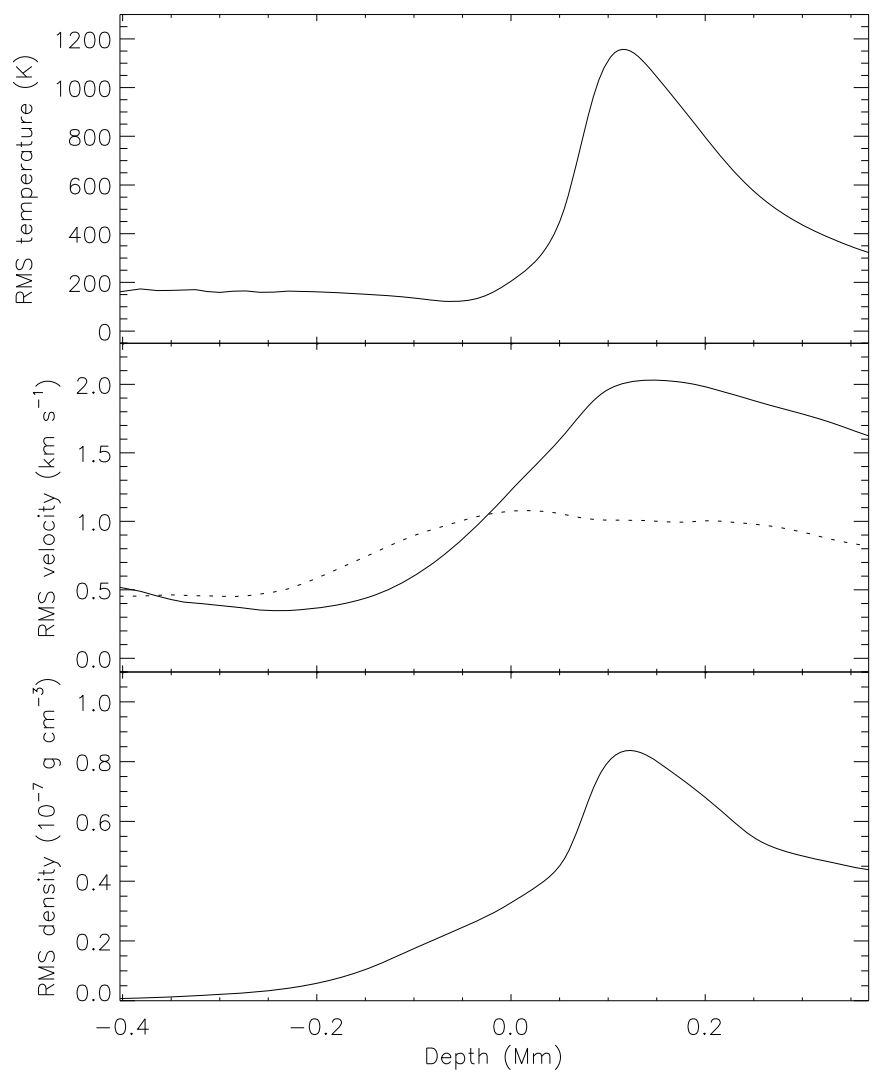

Fig. 6. Root mean square values of the temperature, velocity, and density fields according to our 3D model atmosphere (all snapshots included). In the middle panel, the solid line corresponds to the vertical component of the velocity while the dotted line corresponds to the horizontal component.

abundances, namely $A_{\mathrm{Fe}}$ and $A_{\mathrm{Fe}} \pm 0.5 \operatorname{dex}\left(A_{\mathrm{X}}\right.$ represents the number density of $\mathrm{X}$ relative to hydrogen in a logarithmic scale where the hydrogen abundance is defined as $A_{\mathrm{H}}=12$ ). The adopted iron abundance was solar, more precisely, $A_{\mathrm{Fe}}=7.45$ (Asplund et al. 2005).

We calculated line profiles for the $119 \mathrm{Fe}$ I and $13 \mathrm{Fe}$ II lines used by Ramírez et al. (2007), which are representative of the line-lists adopted in many FGK stellar abundance studies. The sources of atomic data are reliable; transition probabilities have been accurately measured in the laboratory, often by more than one group and averaged after checking for consistency between groups, while the collisional broadening parameters, in particular the van der Waals damping constants, have been obtained from recent theoretical calculations (see Sect. 4.2 in Ramírez et al. 2007 for details and references). To improve the line strength coverage, we added 6 very strong Fe I lines to this list. The atomic data adopted for these strong lines are given in Table 1.

The Doppler shifts introduced by the velocity field of the simulation were taken into account in the synthesis of the absorption line profiles, as well as the thermal and collisional broadening. The microturbulence and macroturbulence parameters are not needed in 3D spectrum synthesis and were therefore not used. 
Table 1. Strong Fe I lines added to the line list of Ramírez et al. (2007) for the $3 \mathrm{D}$ calculation of synthetic line profiles.

\begin{tabular}{ccccc}
\hline \hline $\begin{array}{c}\text { Wavelength } \\
(\AA)\end{array}$ & $\log g f^{a}$ & $\begin{array}{c}\mathrm{EP} \\
(\mathrm{eV})\end{array}$ & $\begin{array}{c}\sigma^{b} \\
(\mathrm{AU})\end{array}$ & $\alpha^{b}$ \\
\hline 5741.85 & -1.67 & 4.26 & 725 & 0.232 \\
6252.56 & -1.77 & 2.40 & 326 & 0.245 \\
6335.32 & -2.18 & 2.20 & 275 & 0.261 \\
6411.65 & -0.72 & 3.65 & 820 & 0.247 \\
6430.85 & -1.95 & 2.18 & 272 & 0.257 \\
6677.99 & -1.42 & 2.69 & 313 & 0.268 \\
\hline
\end{tabular}

${ }^{a}$ Transition probabilities are from the laboratory measurements of the Oxford group (e.g., Blackwell et al. 1976). ${ }^{b}$ The last two columns are the van der Waals damping constants obtained by Barklem et al. (2000); $\sigma$ is the broadening cross section for an atom-perturber relative velocity $v_{0}=10^{6} \mathrm{~cm} \mathrm{~s}^{-1}$, given in atomic units $\left(1 \mathrm{AU}=2.8 \times 10^{-17} \mathrm{~cm}^{2}\right)$, and $\alpha$ the velocity parameter, which is related to the temperature dependence of the cross-section $\left(\sigma \propto T^{(1-\alpha) / 2}\right)$.

\section{Disk-averaged line profiles}

\subsection{Calculation of flux profiles}

The calculation of flux from intensities is straightforward, and very accurate provided enough rays ( $\mu$ and $\phi$ angles) are included in the integrations. The rotationally broadened intensity is

$I(\Delta v, \mu, \phi, V \sin i)=\frac{1}{2 \pi} \int_{0}^{2 \pi} I\left(\Delta v-V \sin i \sin \theta \cos \phi^{\prime}, \mu, \phi\right) \mathrm{d} \phi^{\prime}$

where $V \sin i$ is the projected rotational velocity of the star, and $\phi^{\prime}$ the latitude angle on the stellar disk. The frequency dependence of the intensity has been replaced here with a velocity variable $\Delta v=c \Delta v / v$. The disk averaged line profile is then

$F(\Delta v, V \sin i)=\int_{0}^{2 \pi} \int_{0}^{1} I(\Delta v, \mu, \phi, V \sin i) \mu \mathrm{d} \mu \mathrm{d} \phi$.

\subsection{Bisectors and wavelength shifts of $V \sin i=0$ line profiles}

We used Eqs. (1) and (2) to determine disk-averaged line profiles adopting $V \sin i=0$. The bisectors of these line profiles (Fe I lines only) are shown in Fig. 7, where they have been grouped according to line-depth and excitation potential (EP).

According to the 3D model, the line bisector $\operatorname{span}^{3}$ ranges from about $250 \mathrm{~m} \mathrm{~s}^{-1}$ for the strongest $\mathrm{Fe}$ I lines to about $10 \mathrm{~m} \mathrm{~s}^{-1}$ for the weakest features. The line bisectors show the characteristic $\mathrm{C}$-shape attributed to granulation. The core wavelengths of the weak Fe I features are shifted by up to about $-200 \mathrm{~m} \mathrm{~s}^{-1}$. The blueshift decreases for stronger lines and it becomes nearly zero for features of residual core flux around 0.3. Interestingly, the 3D model predicts a core wavelength redshift for the strongest features, which amounts up to $100 \mathrm{~m} \mathrm{~s}^{-1}$. This result is discussed in more detail below. The core wavelength shifts are also given as a function of the line equivalent width $(E W)$ in Fig. 8.

The large dispersion present between bisectors of different lines that have similar line strength, as shown in Fig. 7, is mostly due to the EP dependence of the core wavelength shifts. Note, for example, that lines of $E W \simeq 50 \mathrm{~m} \AA$ have a shift that is about $30 \mathrm{~m} \mathrm{~s}^{-1}$ higher for the low EP lines compared to those that correspond to the high EP lines, as shown in Fig. 8. Due to this

\footnotetext{
3 We define the "span" as the difference in velocity between the reddest and bluest points of the line bisector.
}

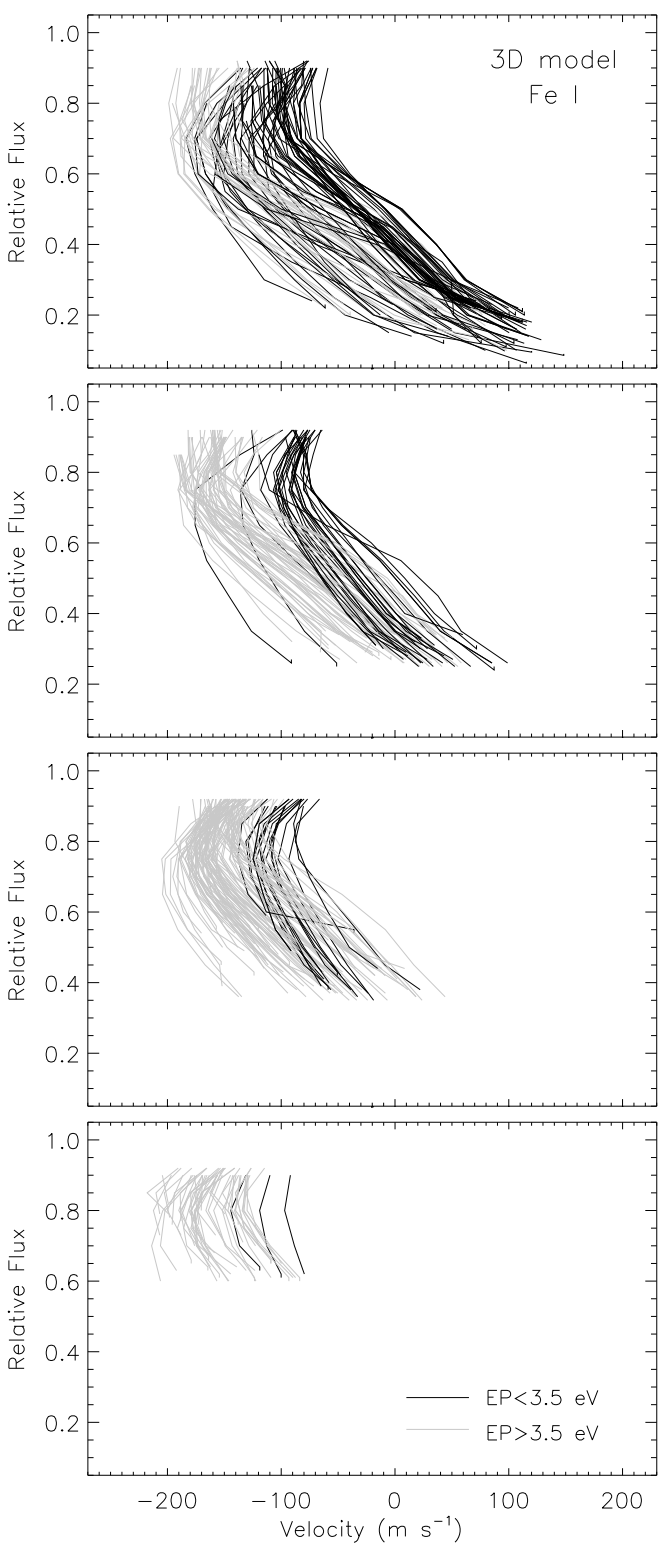

Fig. 7. Bisectors of the disk-averaged Fe I line profiles calculated with our 3D model. Four groups of lines, sorted according to their line-depth, are shown in the different panels. The line bisectors have also been divided into two groups according to the value of their excitation potential (see legend on the bottom panel).

effect, there appear to be two branches in the line shift vs. EW relations shown in Fig. 8, which is, however, due to the fact that our line selection was such that few lines of intermediate EP were included; most of our Fe I lines have either $\mathrm{EP} \simeq 2.5 \mathrm{eV}$ or $\mathrm{EP} \simeq 4.5 \mathrm{eV}$.

When the bisectors of lines of similar strength are compared excluding the core wavelength shifts (therefore comparing only their shapes), the dispersion is reduced significantly, although a small EP dependence for the detailed shapes of the bisectors remains. Qualitatively, while the shape of the lower half of the bisector is nearly independent of EP, for a given line strength, the upper half extends more towards the blue for the high EP lines. Therefore, for a given line depth, the bisector span is slightly larger for higher EP lines. 


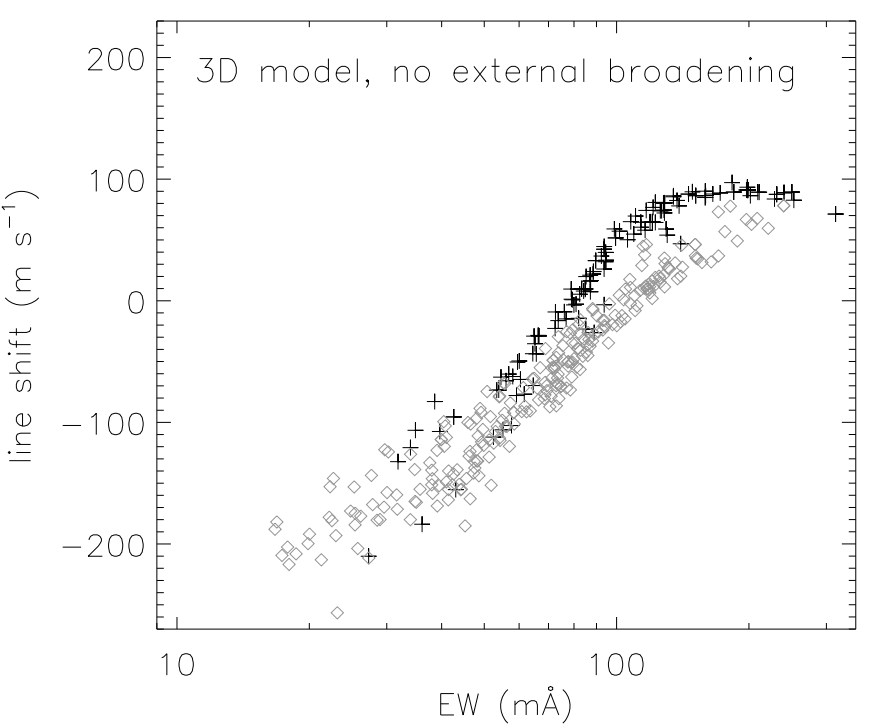

Fig. 8. Core wavelength shifts predicted by our K-dwarf 3D model atmosphere. The Fe I lines have been grouped into low excitation potential $(\mathrm{EP}<3.5 \mathrm{eV}$, black crosses) and high excitation potential (EP $>3.5 \mathrm{eV}$, gray diamonds) lines.

Figure 8 also shows the characteristic signature of granulation. The weakest lines, those that are formed in deep photospheric layers that experience the largest granulation effects, have the highest (in absolute value) core wavelength shifts. As the lines get stronger, and therefore the line formation depth decreases reaching higher photospheric layers with weaker granulation contrasts, the core wavelength shifts become lower.

Since the correlation between velocity and temperature fields decreases as one reaches higher atmospheric layers (see the cyan dots in Fig. 9), one would expect the core wavelength shift of the strongest lines to converge towards zero. Even if the properties of some high photospheric layer are such that most of the light emitted there comes from downflows (as also suggested by Fig. 9; see green dots and blue line), we would expect the redshift to decrease shortly after, provided we continue probing higher layers with stronger absorption lines. A hint of this effect is seen in Fig. 8, where at $E W \simeq 180 \mathrm{~m} \AA$ the line shift, which is already a redshift, seems to start decreasing for higher $E W$ values.

In their study of line formation in solar granulation, Asplund et al. (2000b) encountered a problem for these predicted convective redshifts (see Figs. 11 and 12 in their paper). For the strongest $\mathrm{Fe} I$ features, they are up to $200 \mathrm{~m} \mathrm{~s}^{-1}$ higher than the observed ones. Since the wavelengths of the solar spectrum have been accurately calibrated in an absolute sense and, in particular, no correlation between the errors in the wavelength scale and the line strength are expected in the solar atlases, this may suggest that the predicted core wavelength redshifts for the strongest lines are a numerical artifact, possibly due to deficiencies in the modeling of the outer boundary (Asplund et al. 2000a,b). A test was performed to support this claim and is described in the next paragraph. Note, however, that non-LTE effects and the presence of the chromosphere in real stars may be the dominant factors in determining the absolute core wavelength shifts of those lines, which would make these particular 3D model predictions merely incomplete and not necessarily incorrect. In fact, observations of the solar photosphere show

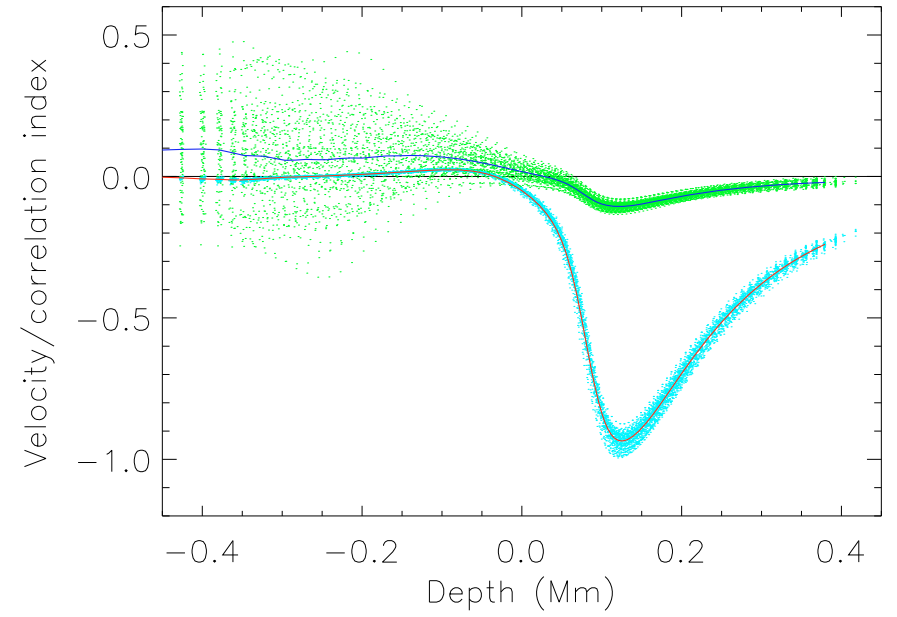

Fig. 9. Velocity and temperature-velocity correlation indices as a function of depth in our $3 \mathrm{~K}$-dwarf model atmosphere (all 100 snapshots are shown here). The velocity index (green dots) is defined as $\overline{v_{z}} / \sigma\left(v_{z}\right)$, where $\overline{v_{z}}$ is the average value of the vertical velocity field at a given depth and $\sigma\left(v_{z}\right)$ its standard deviation. The temperature-velocity correlation index (cyan dots) is given by $0.5 n_{\mathrm{g}}^{-1} \sum\left(v_{z}-\overline{v_{z}}\right)(T-\bar{T})$, where $n_{\mathrm{g}}$ is the number of model grid points and $T$ the velocity field at a given depth with a mean value of $\bar{T}$. The blue and red lines correspond to these indices averaged over all snapshots.

that a reversal of the granulation pattern does occur a few hundred kilometers above the visible surface (e.g., Janssen \& Cauzzi 2006), which would explain line core wavelength redshifts. In our K-dwarf model, a weak temperature-velocity correlation (of opposite sign compared to that of the "normal" granulation pattern seen in continuum formation layers) is predicted at about $-0.1 \mathrm{Mm}$ (see red line in Fig. 9).

A subset of $\mathrm{Fe}$ I features from our linelist were recomputed after excluding a number of upper layers (1,2, and 4$)$ from the simulation. Their core wavelength shifts were then calculated and compared to those obtained from the original model. We find that for $\mathrm{Fe}$ I lines of $E W>100 \mathrm{~m} \AA$, the upper layers play an important role in determining the core wavelength shift. For example, for an $E W=130 \mathrm{~m} \AA$ line, removing 1, 2, and 4 layers increased those shifts by about 6,13 , and $24 \mathrm{~m} \mathrm{~s}^{-1}$, respectively. For $\mathrm{Fe}$ I lines of $E W<100 \mathrm{~m} \AA$, however, the upper layers are unimportant regarding the core wavelength shift. The differences in the core wavelengths obtained with the full model and those derived excluding upper layers have a mean value of essentially zero, with an rms scatter of about $1 \mathrm{~m} \mathrm{~s}^{-1}$. These lines are therefore free from systematic errors due to the limited height of the simulation box and reliable to test the line formation of spectral features commonly used in stellar abundance determinations.

For $E W>100 \mathrm{~m} \AA$, the core wavelength redshift diminishes as more layers are added to the line profile calculation. It is tempting to conclude from this that the redshifts of the strongest lines predicted by the 3D model will become lower if more layers are added to the simulation (although admittedly from this test alone it is clear that the correction would be insufficient to explain the $+100 \mathrm{~m} \mathrm{~s}^{-1}$ predicted by the original simulation for some of the strongest Fe I features). However, the impact of the chromosphere on these upper layers in real stars cannot be ignored, which would make our no-chromosphere model for these uppermost layers unrealistic.

Even though these predicted redshifts appear to reveal a limitation of our current 3D models, note that the line strengths are 


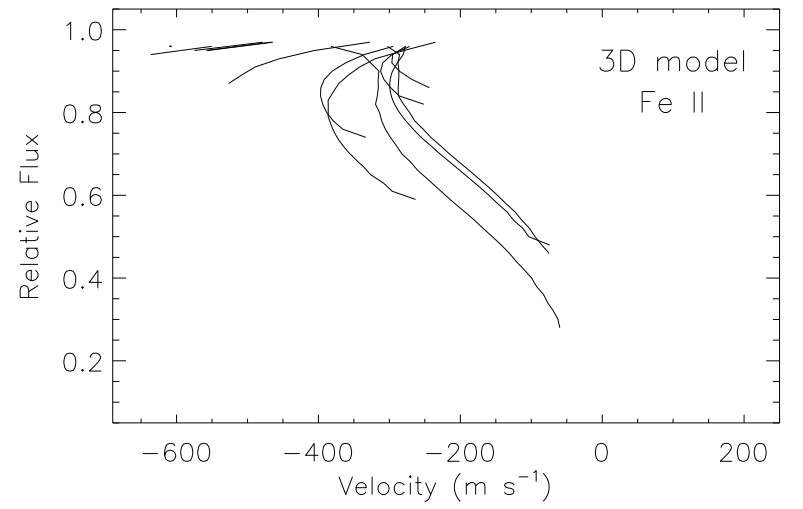

Fig. 10. Bisectors of the disk-averaged Fe II line profiles calculated with our 3D model and assuming $V \sin i=0$.

not affected in the same manner. For example, for the test described above, the corresponding change in equivalent width was between 0 and $1.5 \mathrm{~m} \AA$. For the strongest features, removing 4 of the upper layers resulted in a line equivalent width lower by only about $1 \%$, a number that is comparable to, if not often smaller than, the typical errors in the measurement of line equivalent widths. Thus, the 3D model predictions for the strength of Fe I spectral features of $E W>100 \mathrm{~m} \AA$ are still reliable, given that most of the absorption occurs in layers far from the upper boundary of our 3D model.

Fe II lines suffer from stronger granulation effects. Their bisectors span from about 100 to slightly more than $200 \mathrm{~m} \mathrm{~s}^{-1}$ while the core wavelength blueshifts of the weak lines reach up to about $-600 \mathrm{~m} \mathrm{~s}^{-1}$ (Fig. 10). For $E W$ values between 15 and $40 \mathrm{~m} \AA$, which is the only $E W$ range where our Fe I and Fe II lines overlap, the convective blueshifts of Fe II lines are, on average, about $100 \mathrm{~m} \mathrm{~s}^{-1}$ bluer (i.e., more negative) than those of Fe I lines. This is due, most likely, to the deeper formation depths of Fe II lines. The Fe II number density increases with depth as higher temperatures are required to ionize the neutral iron atom. At these deeper layers, the intensity and velocity fields are strongly correlated. Although it would be ideal to look for the granulation signatures using Fe II lines, in real K-dwarf spectra only a small number of them are available for accurate line profile measurements.

\subsection{Rotationally broadened profiles}

To explore the effect of the projected rotational velocity on line profiles affected by granulation, we computed a few spectral lines using Eqs. (1) and (2) with several non-zero $V \sin i$ values and compared the resultant line bisectors. One typical example of this exercise is shown in Fig. 11.

Between $V \sin i=0$ and $1 \mathrm{~km} \mathrm{~s}^{-1}$, the shape of the line bisector is only slightly affected by stellar rotation and it therefore retains most of the granulation signatures. On the other hand, for projected rotational velocities greater than $1 \mathrm{~km} \mathrm{~s}^{-1}$, the effects of stellar rotation must be taken into account to properly characterize them. In general, the effect of a non-zero $V \sin i$ is to make the line more symmetric. As one might naively expect, additional symmetric broadening dilutes the line asymmetries.

The effect of the rotational velocity on the convective blueshifts is also illustrated in Fig. 11. Interestingly, although the line profiles become more symmetric for higher $V \sin i$ values, the absolute value of the convective blueshift increases. This

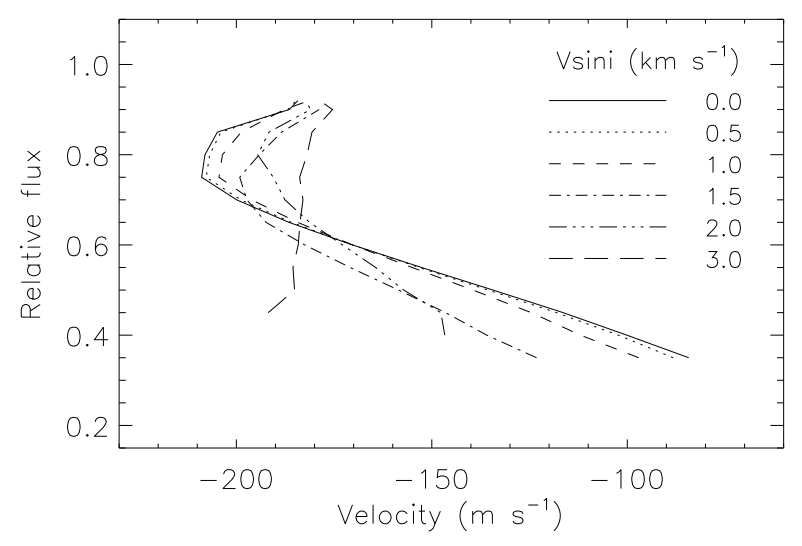

Fig. 11. Bisectors of the theoretical 5228.4 Fe I line computed for several values of the projected rotational velocity $V \sin i$ (see legend).

is a confirmation of the "rotation effect" discussed by Gray \& Toner (1985) and Gray (1986) on the basis of their numerical experiments using two component granulation models. The distribution of Doppler shifts for the granules in a $V \sin i=0$ star extends from a maximum blueshift value corresponding to granules in the disk center to zero for the limb. Since the regions observed near the limb correspond to larger areas due to projection effects, the distribution shows an increase towards lower blueshifts, peaks at a value close to the line-center convective blueshift, and vanishes at zero velocity. A large $V \sin i$ star redistributes the observed Doppler shifts from the granules in areas near the limb symmetrically about zero. Since the distribution does not change for areas near the disk center, the entire disk distribution will now have a red tail but a peak extending further towards the blue, thus producing the rotation effect. Note that this increase in the convective blueshift is an external effect that is not associated with the strength of the granulation inhomogeneities. Rotation is not taken into account in the computation of the model atmosphere, only in the calculation of diskaveraged line profiles from the emergent intensities.

\subsection{Impact of instrumental imperfections}

The observed spectral line profiles have an additional external broadening due to the finite value of the spectral resolution of the spectrograph. In our case (see Paper I for details) we found that the spectral resolution $(R=\lambda / \Delta \lambda)$ was not constant but varied between 160000 and 210000 among our spectra. In addition, we found that the shape of the instrumental profile was slightly asymmetric. Here we explore the impact that these instrumental imperfections have on the theoretical line profiles.

\subsubsection{Variable resolution}

In order to explore the effects of a variable spectral resolution on the theoretical line profiles, we performed tests convolving the disk-averaged, rotationally broadened line profiles with Gaussian instrumental profiles of $F W H M=\lambda / R$. Although the actual instrumental profile of our observations was not Gaussian, its overall shape remained nearly constant (its $F W H M$, however, did change with time). We performed this test with Gaussians to avoid the complications introduced by non-symmetric profiles. The latter is discussed below. We performed the test for 


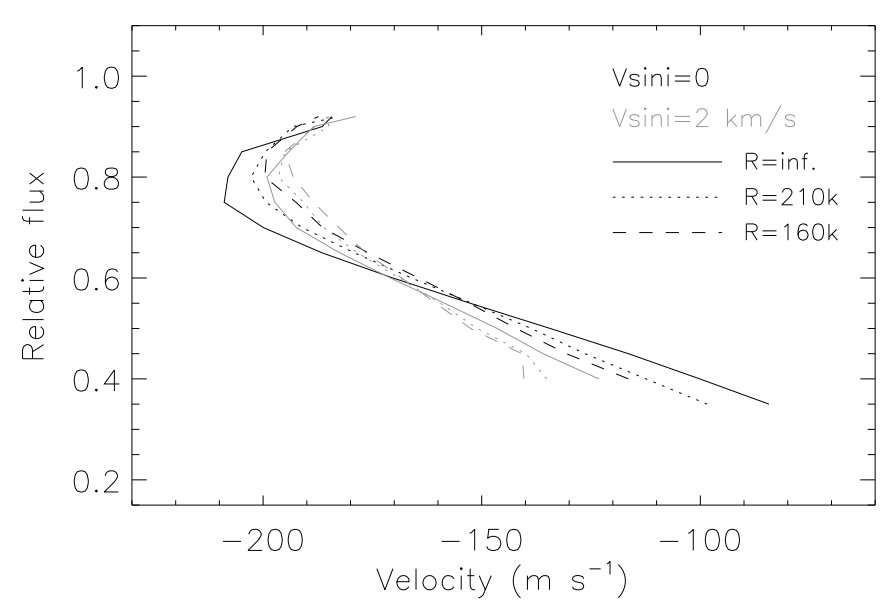

Fig. 12. Bisectors of the theoretical $5228.4 \mathrm{Fe}$ I line computed for $V \sin i=0$ (black lines) and $2 \mathrm{~km} \mathrm{~s}^{-1}$ (gray lines), and three values of the resolving power: infinite (solid lines), $R=210000$ (dotted lines), and $R=160000$ (dashed lines). The instrumental profile was assumed to be a Gaussian for this test.

two values of the projected rotational velocity: $V \sin i=0$ and $2.0 \mathrm{~km} \mathrm{~s}^{-1}$. The result is shown in Fig. 12

As it is clear from inspection of Fig. 12, the effects of a variable resolving power (between 160000 and 210000) on measurements of line asymmetries are relatively small, specially if the profiles have already been rotationally broadened using typical $V \sin i$ values for K-dwarfs. Lower resolution makes the lines more symmetric but, in our range of resolving power and typical values of projected rotational velocity, the asymmetry is reduced, on average, by only a few meters per second.

Note that lowering the spectral resolution using Gaussian instrumental profiles results in an increased core wavelength blueshift. This is due to the fact that the lines are asymmetric and the asymmetry is such that the blue wing extends more than what is expected for a symmetric profile. In the "blurring" of the spectral line, this extended wing contributes more to the shallowing of the line core than the red wing, resulting in an additional core wavelength blueshift.

\subsubsection{Asymmetric instrumental profile}

In Paper I, we showed that the instrumental profile of our observations was very similar to a Gaussian profile but not a perfect match. The largest departure from Gaussianity was observed on the blue wing, where a small bump was present and affects all our data.

Figure 13 shows how an asymmetric instrumental profile affects the shape of a line bisector as well as its core wavelength shift. This test was made generating a Gaussian profile and introducing small perturbations instead of using the real instrumental profile for practical reasons; the shape of the actual instrumental profile is not as smooth as a Gaussian function due to the noise of the ThAr exposures used to determine it (see Sect. 3.5 in Paper I for details) and it is not as trivial as the Gaussian case to deconvolve a non-smooth profile to take into account thermal broadening, which represents about $5 \%$ of the total broadening of the Th lines (we do use the real instrumental profile later for the comparison with our observations).

Compared to the Gaussian case, our slightly asymmetric instrumental profile affects both the line bisector span and core wavelength shift of the line profiles formed in stellar granulation.

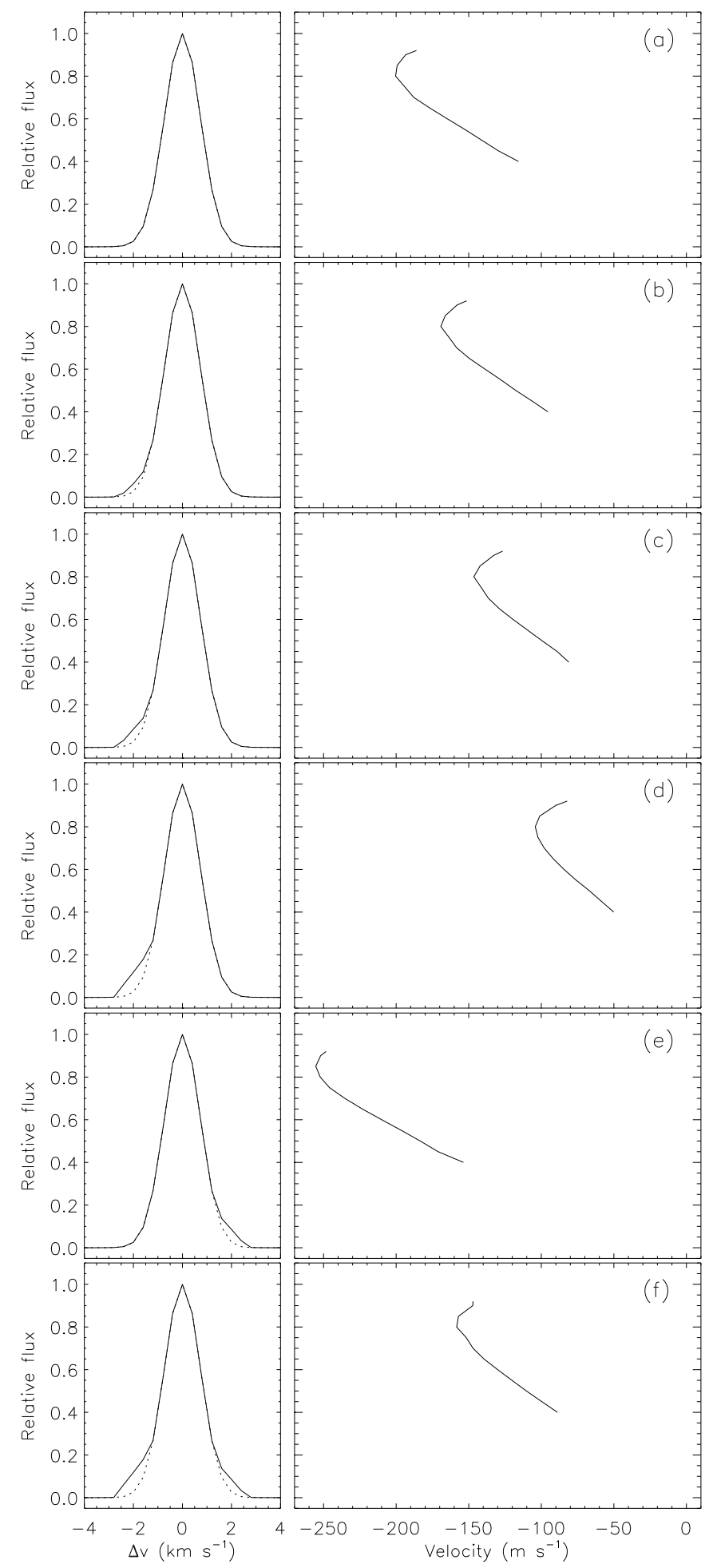

Fig. 13. Effect of an asymmetric instrumental profile (left panels) on the shape and core wavelength shift of the line bisector (right panels). The $5228.4 \AA \mathrm{Fe}$ I line was used here to illustrate the effect. The top panel a) shows the case of a Gaussian instrumental profile.

A blue bump on the profile reduces the span and shifts the entire line towards the red by an amount that depends on the size of the bump, as seen by the sequence (b), (c), (d) in Fig. 13. The size of each of these bumps is such that the area under the instrumental profile compared to the Gaussian case is 1.6 (a), 2.8 (b), and $5 \%$ (c) higher while the shifts are about 20,40, and $70 \mathrm{~m} \mathrm{~s}^{-1}$, 
respectively. Similarly, a red bump that produces a $2.8 \%$ departure from Gaussianity increases the span and introduces an extra blueshift of about $30 \mathrm{~m} \mathrm{~s}^{-1}$, as shown by case (e). Note, however, that the instrumental profile of our observations does not show a red bump alone. Only for one of our observing runs, both a blue and a red bump were present. Panel (f) in Fig. 13 approximately corresponds to that case (here the departure from Gaussianity corresponds to $7.8, \%$ of covered area difference). The instrumental profile of our observations varied with time, but the relative departure from Gaussianity was nearly time-independent. Cases (b), (c), and (f) in Fig. 13 fairly represent our data, with (c) corresponding to the bulk of them (cf. Fig. 4 in Paper I).

Thus, we find that after convolving the predicted line profiles with our asymmetric instrumental profile (represented by cases b, c, and f in Fig. 13), the span of the line bisector is somewhat affected (it is reduced by about $10 \mathrm{~m} \mathrm{~s}^{-1}$ ) while the core wavelength shift changes significantly (about 20-40 $\mathrm{m} \mathrm{s}^{-1}$ towards the red) compared to the Gaussian approximation.

\subsection{Reference mean theoretical line bisectors and wavelength shift vs. EW relation}

For the comparison with observed data, the line profiles predicted by our 3D model should be convolved with appropriate values of rotational and instrumental profiles. Given that these are relatively fine effects for the sample at hand and the fact that only one star in the sample (HIP 86400) has fundamental parameters very close to those of the 3D model, we define a reference case of $V \sin i=1.5 \mathrm{~km} \mathrm{~s}^{-1}$ (roughly the projected rotational velocity of HIP 86400, as shown in Appendix B) and an instrumental profile that corresponds to the observing run when most of the data for HIP 86400 were acquired (see Paper I). Hereafter, we will refer to the theoretical line bisectors and wavelength shifts as those that correspond to this particular choice of external broadening parameters.

The line bisector is an excellent probe of line formation in stellar granulation because at each flux depth its value is sensitive to the inhomogeneities of a certain range of photospheric layers. We thus expect the shapes of bisectors of lines of a given strength to be similar. There will be, of course, differences due to dissimilar transition properties such as oscillator strength, excitation potential (cf. Fig. 7), collisional broadening, etc. Nonetheless, grouping lines of similar strength to obtain mean theoretical line bisectors is necessary in the context of this work. In Paper I we showed that measuring individual line bisectors is a very difficult task and the results very noisy, but averaging bisectors of many lines of similar line strength resulted in very robust mean line bisectors. We thus follow the same procedure with the theoretical results.

The mean theoretical line bisectors for Fe I lines with residual core flux around $0.30,0.45$, and 0.65 are shown in Fig. 14. The error bars shown there (shaded areas) correspond to the 1- $\sigma$ standard deviation from the mean, which is the intrinsic dispersion that we would expect from comparison with observed lines. For clarity, Fig. 14 shows the line bisectors measured with respect to the line center. The core wavelength shifts are shown separately in Fig. 15. We perform this separation because the observed data is treated in that way to avoid introducing the error of the rest core wavelengths in the determination of the shapes of the mean line bisectors.

For comparison, Fig. 14 also shows the mean line bisectors that are obtained from the $V \sin i=0$ profiles without instrumental broadening (dotted lines) and also those obtained from the $V \sin i=1.5 \mathrm{~km} \mathrm{~s}^{-1}$ profiles convolved with a Gaussian
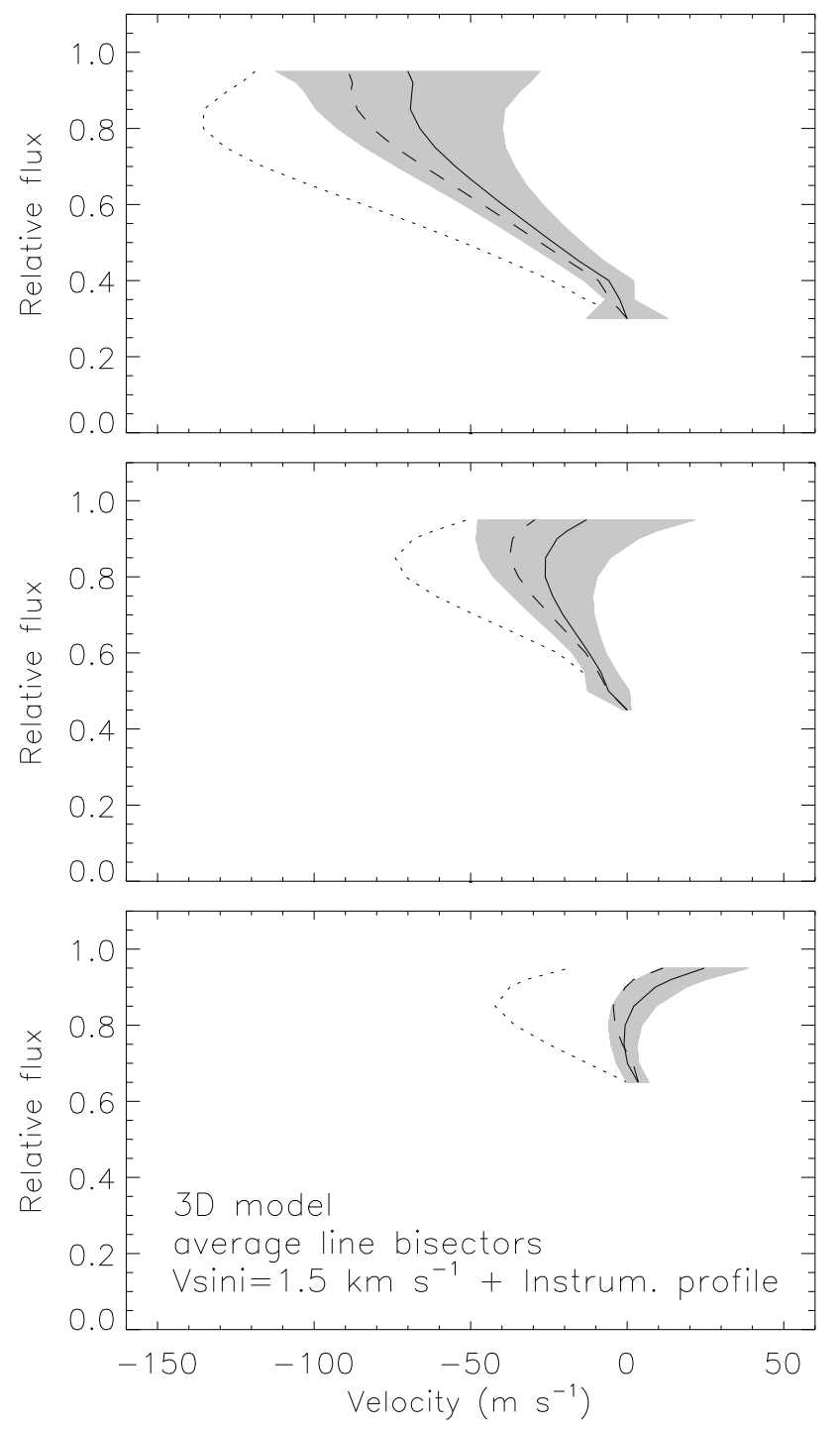

Fig. 14. Solid lines: mean line bisectors obtained from the theoretical line profiles computed with our 3D model and adopting $V \sin i=$ $1.5 \mathrm{~km} \mathrm{~s}^{-1}$ and an (asymmetric) instrumental profile corresponding to our observations of the reference star HIP 86400 . The shaded areas correspond to the 1- $\sigma$ dispersion of the theoretical line-by-line bisectors. Each bisector has been forced to have zero velocity at line center. The dotted lines show the mean theoretical line bisectors assuming $V \sin i=0$ and no instrumental broadening while the dashed line shows the same mean bisectors but adopting a Gaussian instrumental profile of $R \simeq 180000$.

instrumental profiles of $F W H M$ equal to that of the real instrumental profile ( $R \simeq 180000$; dashed lines). Clearly, the effect of the projected rotational velocity on the mean bisector shape is more important than that of the asymmetry of the instrumental profile, although the impact of the latter is not negligible, in particular for the regions near the continuum.

The core wavelength shift vs. $E W$ relation that results from the line profiles calculated with our 3D model, after convolution with the reference rotational and instrumental profiles, is shown in Fig. 15. This figure should be compared to Fig. 8, which shows the corresponding result for $V \sin i=0$ and no instrumental broadening. In addition to vary slower, the reference line shift vs. $E W$ relation (Fig. 15) is shifted upwards due to the effects of the asymmetric instrumental profile (Sect. 4.4.2), which 


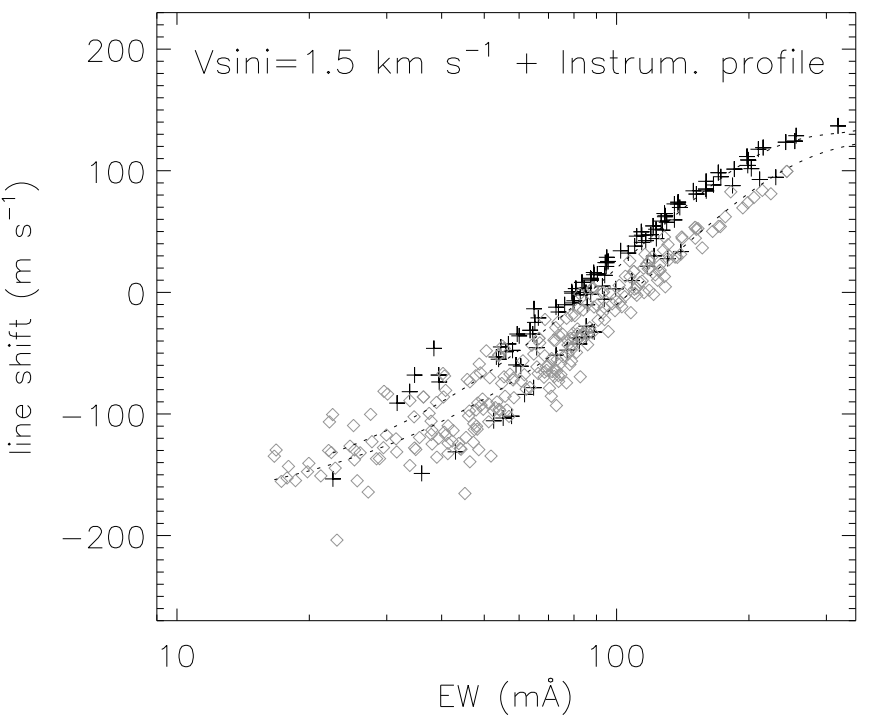

Fig. 15. Core wavelength shifts obtained from the theoretical line profiles computed with our 3D model and adopting $V \sin i=1.5 \mathrm{~km} \mathrm{~s}^{-1}$ and an (asymmetric) instrumental profile corresponding to our observations of the reference star HIP 86400 . The Fe I lines have been grouped into low excitation potential (EP $<3.5 \mathrm{eV}$, black crosses) and high excitation potential (EP $>3.5 \mathrm{eV}$, gray diamonds) lines. The dotted lines are cubic fits to the predicted line shifts of the two EP groups.

in our case is more important than the rotation effect that shifts the lines towards the blue instead (Sect. 4.3).

In Fig. 15, the lines have been separated into high (EP > $3.5 \mathrm{eV})$ and low $(\mathrm{EP}<3.5 \mathrm{eV})$ excitation potential lines, as was done in Sect. 4.2. Two independent cubic polynomial fits were made, one for each group, and are shown with the dotted lines in Fig. 15. These fits are used below for the comparison with the observed data.

\section{Comparison to observations}

In Paper I, the very high spectral resolution, high signal-to-noise ratio spectra that we obtained for nine bright K-type dwarf stars were described in detail. Here those data are used to compare the predictions of our hydrodynamic model atmosphere and 3D spectrum synthesis with the observed shapes of Fe I absorption line profiles and thus verify the accuracy of the model. In Figs. 16 and 17 we show the comparison of bisectors and wavelength shifts, respectively, for four of our sample stars.

\subsection{Line bisectors}

In general, the basic observed shapes of the line bisectors are well reproduced by the model. Quantitatively, however, there are measurable differences. In Fig. 16, three average spectral line bisectors are shown in each panel. The one representing strong (weak) lines was obtained with features of line depth between 0.25 and $0.40(0.60$ and 0.80$)$, which roughly corresponds to $E W=80-140 \mathrm{~m} \AA(20-40 \mathrm{~m} \AA)$. The intermediate strength bisector corresponds to all other lines in between (i.e., line depth between 0.4 and 0.6 and $E W=40-80 \mathrm{~m} \AA$ ).

The mean line bisectors of HIP $96100\left(T_{\text {eff }} \simeq 5220 \mathrm{~K}\right)$ have clearly larger span than those predicted by the 3D model (see the top panel in Fig. 16). There are three reasons that can explain this. First, the effective temperature of this star is about $400 \mathrm{~K}$

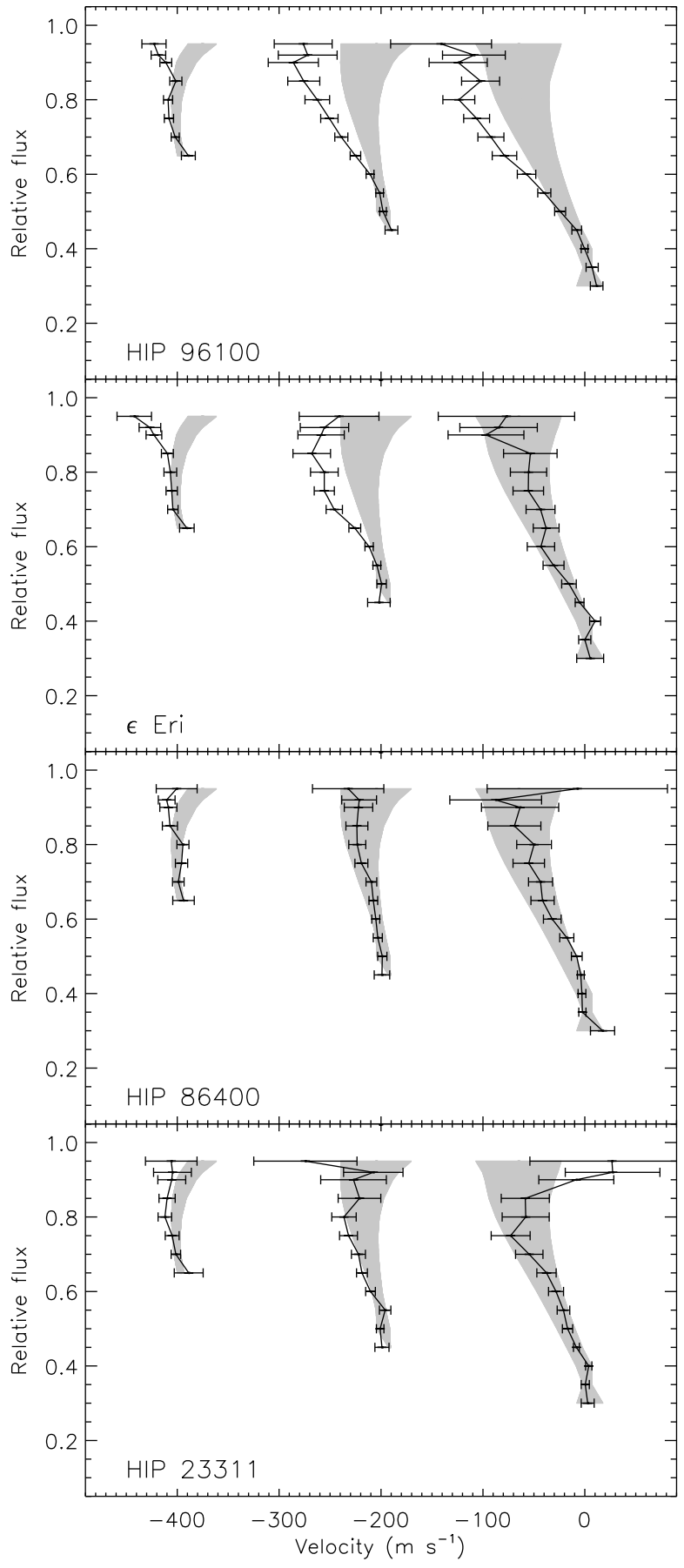

Fig. 16. Comparison of theoretical and observed mean Fe I line bisectors for four K-dwarfs. These bisectors are measured with respect to the line core wavelengths, arbitrary shifts of -200 and $-400 \mathrm{~m} \mathrm{~s}^{-1}$ have been applied to the weaker lines for clarity. The theoretical bisectors are represented by the gray shaded areas; their extent corresponds to the 1$\sigma$ intrinsic scatter that results from the averaging of several Fe I line profiles. The solid lines with error bars correspond to the observations (see Paper I for details).

hotter than the 3D model, second, its metallicity is lower than solar $([\mathrm{Fe} / \mathrm{H}]=-0.22)$, and, finally, its projected rotational velocity is the lowest among our sample $\operatorname{stars}(V \sin i \simeq$ $\left.0.8 \mathrm{~km} \mathrm{~s}^{-1}\right)^{4}$. Note that the granulation effects, and in fact the

\footnotetext{
4 For more details and references on the stellar $V \sin i$ values reported in this paper, see Table A.1 in Paper I.
} 
absolute magnitude of the inhomogeneities, are argued to increase with effective temperature (Sect. 2) and also with decreasing metallicity, although probably the latter effect is significantly smaller (e.g., Allende Prieto et al. 1999), while low projected rotational velocities $\left(V \sin i<1 \mathrm{~km} \mathrm{~s}^{-1}\right.$ ) were shown not to affect the shape of the line bisectors significantly (Fig. 11).

In this context, it is interesting to see the case of $\epsilon$ Eri $\left(T_{\text {eff }} \simeq 5050 \mathrm{~K}\right)$, which is more than $200 \mathrm{~K}$ warmer than the $3 \mathrm{D}$ model and yet does not show bisectors with a span significantly larger than the 3D model predictions, in particular for the strong line case. Part of the reason for this is the high $V \sin i$ value of the star (approximately $2 \mathrm{~km} \mathrm{~s}^{-1}$ ), which, as seen in Fig. 11, can reduce somewhat the span of the line bisectors. Although it is not shown in Fig. 16, a similar result is found for the stars HIP 26779, HIP 88 601, HIP 64 797, and HIP 37349 , which are also hotter than the 3D model by 50 to $300 \mathrm{~K}$ and have high $V \sin i$ values (between 2 and $3 \mathrm{~km} \mathrm{~s}^{-1}$ ). Interestingly, the 5 stars mentioned above are also the most active in our sample (cf. Fig. B.1 in Paper I), which suggests that chromospheric activity or some other activity-related effect has an important impact on the shapes of Fe I line profiles.

HIP $86400\left(T_{\text {eff }} \simeq 4830 \mathrm{~K},[\mathrm{Fe} / \mathrm{H}]=-0.05\right)$ is our reference star because it has parameters very close to those adopted in the calculation of the 3D model atmosphere. A visual inspection of the third panel in Fig. 16 shows that the agreement between the observed and predicted mean line bisectors is excellent. The line bisector spans for the strongest Fe I features in our HIP 86400 spectrum are about $70 \mathrm{~m} \mathrm{~s}^{-1}$. Since this value agrees very well with the theoretical prediction, after accurately taking into account the effects of finite spectral resolution, detailed instrumental profile, and projected rotational velocity, we can conclude that the actual bisector spans in this type of star (solar-metallicity early K-dwarfs) are around $140 \mathrm{~m} \mathrm{~s}^{-1}$ for the strongest Fe I features. The exact observed value will of course depend on the properties of the instrument and the value of the projected rotational velocity of the star.

The coolest stars in our sample, HIP $114622\left(T_{\text {eff }} \simeq 4740 \mathrm{~K}\right)$ and HIP 23311 ( $\left.T_{\text {eff }} \simeq 4640 \mathrm{~K}\right)$, have both relatively low $V \sin i\left(\lesssim 2 \mathrm{~km} \mathrm{~s}^{-1}\right)$ and are about 80 and $180 \mathrm{~K}$ cooler than our $3 \mathrm{D}$ model. Their spectra are richer in spectral lines compared to those of our other sample stars and this is likely the reason why their mean bisectors show large deviations from the theoretical expectation near the continuum (the bottom panel of Fig. 16 shows only the results for HIP 23311, which are very similar to those of HIP 114622). In fact, the mean bisectors approach zero velocity there, which points to the influence of numerous blends randomly distributed on both sides of the spectral lines. Nonetheless, farther from the continuum, the 3D model predictions agree quite well with the observations of these two cooler stars. Therefore, it is likely that the granulation contrast and convective velocities do not change significantly in dwarf stars of effective temperature between 4600 and $4800 \mathrm{~K}$.

A comment should be added here about the theoretical mean bisector of the strongest lines. Since their core wavelengths are shifted by up to $+100 \mathrm{~m} \mathrm{~s}^{-1}$, and we concluded this to be a limitation of our model (Sect. 4.2), the lower part of their line bisectors should be similarly affected. The strongest features that were used to determine the mean line bisectors shown in Fig. 16 have $E W \simeq 140 \mathrm{~m} \AA$, which is higher than the upper limit for the reliability of our $3 \mathrm{D}$ model results $(E W \simeq 100 \mathrm{~m} \AA)$. The bisectors of $E W \simeq 140 \mathrm{~m} \AA \mathrm{Fe}$ I lines are well represented in the second panel of Fig. 7. Note that the low EP lines are the most affected by the incorrect redshifts predicted by our 3D model whereas the high EP lines have, on average, zero shift. When plotted with respect to their core wavelengths (i.e., shifted so that the lowest flux pixel is at zero velocity), low and high EP lines show somewhat similar bisectors in this group of lines. Interestingly, of all observed lines used to construct Fig. 16, about $80 \%$ have high EP $(>3.5 \mathrm{eV})$. Thus, this comparison between theory and observation is mostly unaffected by the model uncertainties because, for this group of lines, both high and low EP lines show similar relative line bisectors (therefore the theoretical average strong line bisector shown in Fig. 16 does not change significantly if computed separately for each EP group) and the observations are dominated by high EP lines, which, when computed with our 3D model, are less affected, in an absolute sense, by these limitations.

\subsection{Wavelength shifts}

The line core wavelength shift vs. equivalent width relations for four of our sample stars are shown in Fig. 17, along with the model predictions. In this figure, we have forced the average observed velocity for the strongest lines $(E W \gtrsim 100 \mathrm{~m} \AA)$ to be zero (cf. Paper I). The basic signature of granulation is evident in all cases and it is reasonably consistent between theory and observation, as long as we consider lines of $E W<100 \mathrm{~m} \AA$, given that the theoretical predictions for the line shifts are uncertain at large $E W$ (Sect. 4.2).

For HIP 96 100, the hottest star of the sample, a visual inspection of Fig. 17 shows that the blueshifts are underestimated by the model below $E W \simeq 100 \mathrm{~m} \AA$. This is most likely due to the higher effective temperature of the star compared to that of the model. In general, we expect the slope in this relation to increase with increasing temperature.

The observed line shift vs. equivalent width relation for $\epsilon$ Eri is more shallow than that of the 3D model and perhaps even more shallow also than those of the cooler stars shown in Fig. 17. In addition, there is significant scatter, even though this is the brightest star of the sample and we expect random errors to be minimum (in addition to the larger scatter of the bin-averaged values shown in Fig. 17, the error bars that represent the lineby-line scatter are also larger compared to those of fainter stars). These properties are also seen in other high $V \sin i$ stars with clear signatures of strong chromospheric activity in our sample (these stars are not shown in Fig. 17 but in this context they behave similarly to $\epsilon$ Eri). Interestingly, stellar activity has been shown to affect the shapes of the line cores significantly (e.g., Gray 1984; Borrero 2008), which explains our results, at least qualitatively.

For HIP 86400 , the reference star, the agreement between model and observation is excellent for lines of $E W<100 \mathrm{~m} \AA$. The predicted slope of the relation for weak lines is remarkably similar to that given by the observational data. The maximum observed convective blueshift is about $-150 \mathrm{~m} \mathrm{~s}^{-1}$. Note that the theoretical relation does not flatten completely below $100 \mathrm{~m} \AA$ but it seems to become slightly less dependent on $E W$ for the weakest lines $E W<40 \mathrm{~m} \AA$. This is also in very good agreement with the observations, even though blends introduce significant error to our measurements for the weakest lines.

The agreement is also good for the cooler stars HIP 114622 and HIP 23311 (not shown), confirming our suspicion that the characteristics of granulation are quite similar between these two stars and HIP 86400 , despite the $\sim 200 \mathrm{~K}$ difference in $T_{\text {eff }}$. 


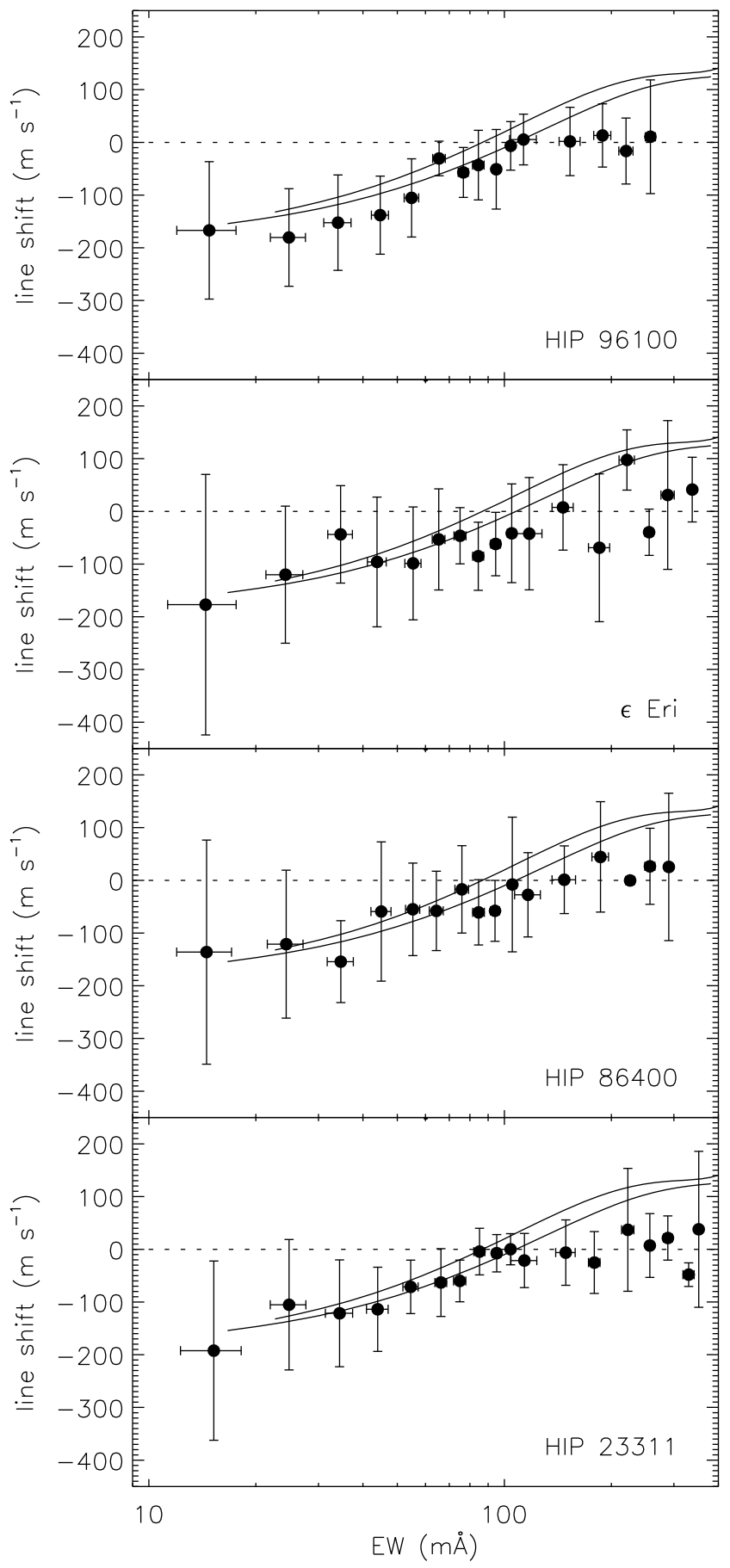

Fig. 17. Comparison of theoretical and observed line core wavelength shift vs. $E W$ relation for four K-dwarfs. The observed data (filled circles with error bars) represent average values in bins of equivalent width and have been shifted so that the average velocity of the strongest lines $(E W \gtrsim 100 \mathrm{~m} \AA$ ) is zero (see Paper I for details). The solid lines correspond to the predictions of the 3D model (cf. Fig 15). The two solid lines correspond to the relations obtained for low and high EP lines, as explained in Sect. 4.5. Note that the $x$-axis scale is logarithmic. The dotted line corresponds to zero line core wavelength shift.

\section{Conclusions}

A three dimensional radiative-hydrodynamical simulation has been computed for stellar parameters $T_{\text {eff }}=4820 \mathrm{~K}, \log g=4.5$, and $[\mathrm{Fe} / \mathrm{H}]=0$, using the same prescription that has been successful at reproducing the granulation features in the Sun. This
K-dwarf model also predicts a granulation pattern, i.e., a correlation between the intensity (or temperature), velocity, and density fields, which is, however, weaker than that found for hotter stars. Strong temperature contrasts at a given height occur well below the visible surface, contrary to the case of hotter stars where this happens nearer their visible surface, but the velocity field still shows significant fluctuations in high photospheric layers.

The 3D model calculations for typical K-dwarfs show a span in the theoretical line bisectors between about 10 and $250 \mathrm{~m} \mathrm{~s}^{-1}$, depending on line strength, while the maximum core wavelength shifts (convective blueshifts) are about $-200 \mathrm{~m} \mathrm{~s}^{-1}$ for the Fe I lines and $-600 \mathrm{~m} \mathrm{~s}^{-1}$ for the Fe II lines.

Line broadening due to the projected rotational velocity of stars must be taken into account when comparing the model predictions with observational data. The impact of the finite resolving power of our observations (presented in the first part of this series) was carefully analyzed and taken into account before comparing theory to observations. We explored the effect of both a variable $F W H M$ and an asymmetric instrumental profile. The former has only a small impact on the line bisectors and wavelength shifts (within the range of spectral resolution of this work) but the latter produce significant additional shifts to the core wavelengths of the lines.

Although there is a possibility that other effects, for example those related to stellar activity, modify the detailed shapes of spectral lines profiles, the agreement between the 3D model predictions and the observations is satisfactory. If there are effects other than granulation affecting the line profiles, we expect their impact to be smaller.

This good agreement demonstrates that our 3D model for K-dwarf granulation accounts for most of the factors that determine the detailed shapes of Fe I line profiles, as well as their strengths, as quantified by, for example, the line equivalent width. This, in turn, means that the physics invoked in these parameter-free simulations is fairly realistic. Our model is thus adequate to explore 3D effects on chemical abundance studies, which will be done in Paper III.

It should be emphasized that even though our 3D model represents significant progress relative to the $1 \mathrm{D}$ scenario, there is still room for improvement. For example, the simulation predicts convective redshifts of about $100 \mathrm{~m} \mathrm{~s}^{-1}$ for the strongest Fe I lines, but implications based on solar observations and their comparison to our K-dwarf data indicate that they should be nearly zero. Non-LTE effects, the chromosphere, or numerical artifacts associated with our 3D model calculations could be responsible for this discrepancy.

Acknowledgements. This work was supported in part by the Robert A. Welch Foundation of Houston, Texas. The authors acknowledge the Texas Advanced Computing Center (TACC) at The University of Texas at Austin for providing HPC resources that have contributed to the research results reported within this paper (URL: http: //wwW . tacc . utexas . edu).

\section{Appendix A: The advanced spectrum synthesis 3D tool (Asset)}

A computer code for solving the radiative transfer problem in 3D model atmospheres has been recently developed by Koesterke et al. (2008) and was made available for this work.

Koesterke et al. (2008) "Advanced Spectrum Synthesis 3D Tool" (Ass $\epsilon$ t) represents an improvement over previous 3D/LTE codes because it is not limited to the calculation of single lines, simple blends, or constant background opacities. Also, it properly includes electron and Rayleigh scattering due to atomic hydrogen, and uses improved (higher-order) interpolation schemes 

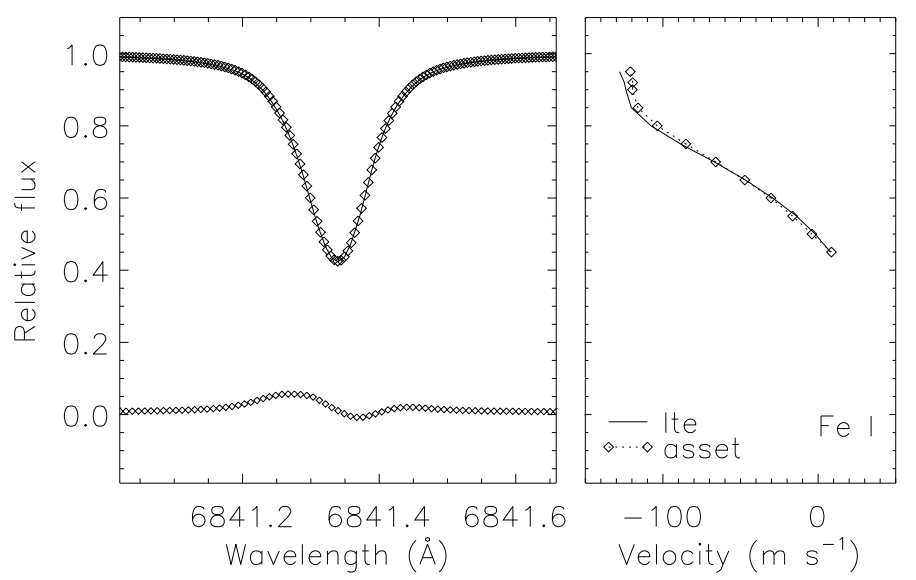

Fig. A.1. Left: Spectral lines synthesized with the 3D codes 1te (solid line) and Asset (diamonds). Their difference (lte minus Asset) is shown at the bottom, amplified by a factor of 10. Right: Bisectors of the lines shown on the left panel.

within the simulation grid points for the calculation of opacities and intensities. Asset is also capable of working with 1D models, which allows $1 \mathrm{D}$ vs. 3D comparisons to be made in a consistent manner. Furthermore, the opacity calculation is based on a modified version of the thoroughly tested 1D code SYNSPEC (Hubeny 1988; Hubeny \& Lanz 1995).

In Paper III, we will use Asset to calculate molecular and atomic features as well as the continuum over wide spectral windows. For the synthesis of atomic lines in this paper, however, we used the 3D code "1te" (e.g., Asplund et al. 2000b), as described in Sect. 3. We computed 23 of the spectral lines used in this paper (17 Fe I and $6 \mathrm{Fe}$ II) with Asset to perform a code-tocode comparison. Input data were carefully checked for consistency. A typical result of the comparison of detailed line profiles is shown in Fig. A.1 while the differences in the predicted equivalent widths and core wavelength shifts are shown in Fig. A.2.

In general, the wings of line profiles computed with lte are slightly deeper, in particular the blue wing. This makes the line bisector span slightly larger, by less than about $10 \mathrm{~m} \mathrm{~s}^{-1}$ for the example shown in Fig. A.1. This also leads to a slightly higher (more negative) convective blueshift for the lte case, as shown in the middle panel of Fig. A.2. The line core blueshift is higher (more negative, by about $15 \mathrm{~m} \mathrm{~s}^{-1}$ ) according to lte.

The top panel of Fig. A.2 shows that the equivalent widths obtained from the line profiles computed with both codes agree within $1 \mathrm{~m} \AA$ below $90 \mathrm{~m} \AA$ and the difference shows a small trend for the strongest lines, with the $E W$ values from lte being lower by at most $2 \mathrm{~m} \AA$. These differences can be attributed not only to the slightly different detailed shapes of the profiles but also to the continuum level computed for each case.

Note that the line shift vs. $E W$ relation is only slightly affected by the choice of 3D spectrum synthesis code (bottom panel of Fig. A.2). Only a small shift upwards of $15 \mathrm{~m} \mathrm{~s}^{-1}$ should be considered if Asset is used instead of lte. Since both the line bisectors and line shift vs. $E W$ relation are nearly independent on the synthesis code adopted, our conclusions about the good agreement between theory and observation presented in Sect. 5 are robust.

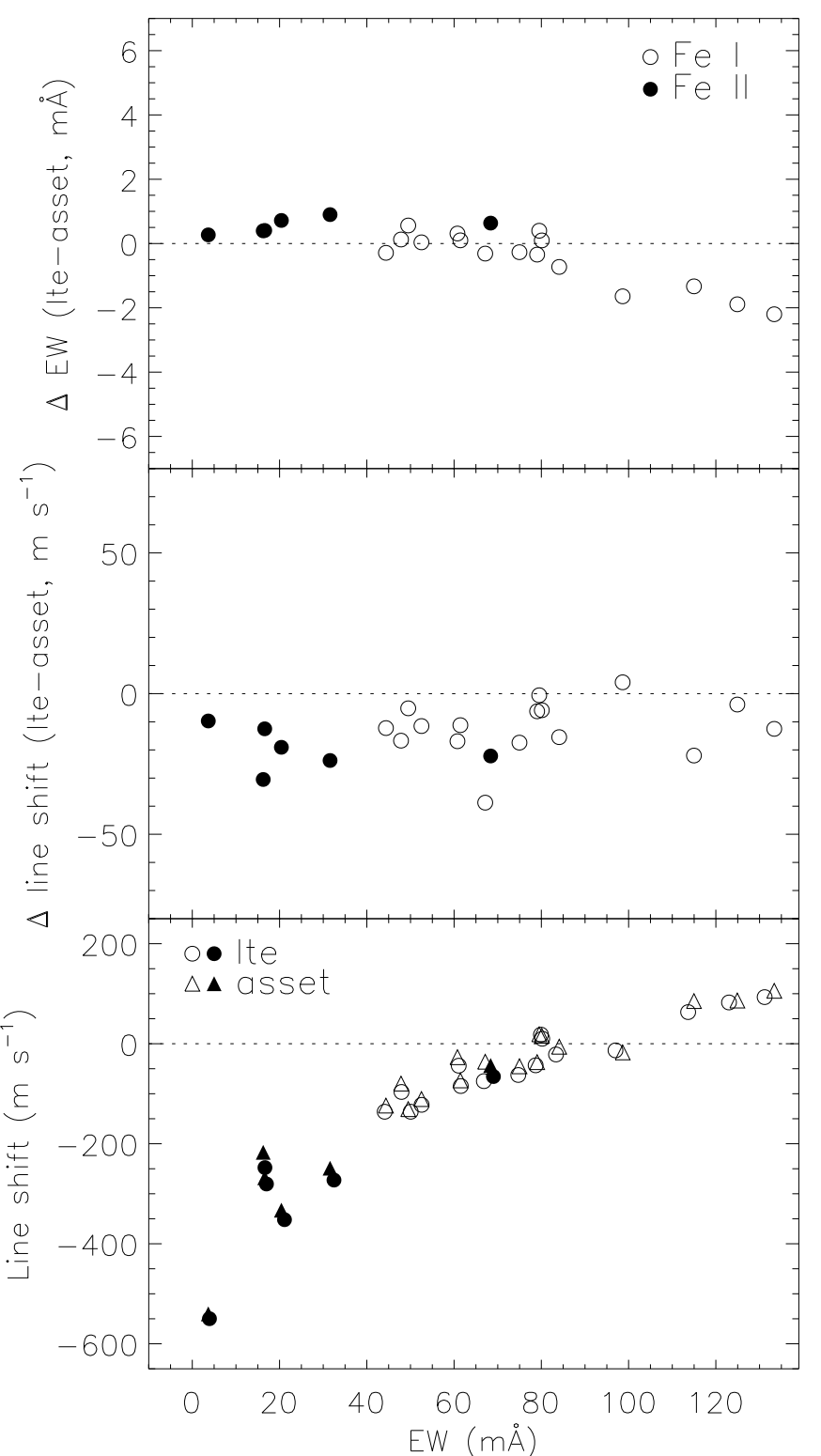

Fig. A.2. Difference in equivalent width (top panel), line shift (middle panel), and $E W$ vs. line shift relation (bottom panel) between the predictions of lte and Asset. Filled (open) symbols correspond to Fe II (Fe I) lines. In the bottom panel, the triangles (circles) correspond to the Asset (lte) predictions.

\section{Appendix B: The projected rotational velocity of HIP 86400}

By convolving line profiles predicted by our 3D model with projected rotational velocity and instrumental profiles, as described in Sect. 4, we determined a more accurate $V \sin i$ value for HIP 86400 . We did not perform similar calculations for the other sample stars because the strength and FWHM of the lines are very sensitive to the stellar parameters, in particular $T_{\text {eff }}$, and only HIP 86400 has parameters identical (within observational errors) to those of our 3D model atmosphere.

We selected 14 of the "cleanest" iron lines available in the HIP 86400 spectrum and computed the difference between observed and predicted profiles around $\pm 0.15 \AA$ from the line center using only two free parameters, the $\log g f$ value (or, 


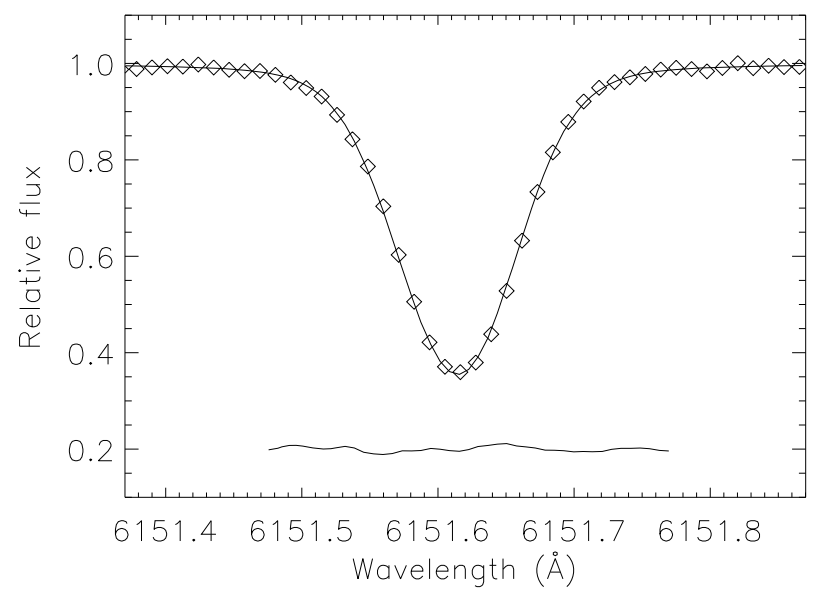

Fig. B.1. Minimum $\chi^{2}$ model fit (solid line) to the observed $6151 \AA$ line profile (diamonds) in our HIP 86400 spectrum. Residuals around $\pm 0.15 \AA$ of the line center are shown at 0.2 relative flux.

equivalently, iron abundance) and $V \sin i$. By minimizing the difference between observed and predicted profiles, using a $\chi^{2}$-like scheme, we determined the $V \sin i$ value of HIP 86400 . No additional broadening, in particular microturbulence or macroturbulence, was necessary to accurately fit the observed line profiles. One of our best fits to the data is illustrated in Fig. B.1. The average of the $V \sin i$ values obtained from all lines, weighted by the quality of each fit, is $V \sin i=1.57 \pm 0.20 \mathrm{~km} \mathrm{~s}^{-1}$ (the error bar corresponds to the 1- $\sigma$ scatter of the line-by-line values).

\section{References}

Allende Prieto, C., García López, R. J., Lambert, D. L., \& Gustafsson, B. 1999, ApJ, 526, 991

Allende Prieto, C., Asplund, M., García López, R. J., \& Lambert, D. L. 2002, ApJ, 567, 544

Asplund, M. 2005, ARA\&A, 43, 481

Asplund, M., Nordlund, Å., Trampedach, R., \& Stein, R. F. 1999, A\&A, 346, L17

Asplund, M., Ludwig, H.-G., Nordlund, Å., \& Stein, R. F. 2000a, A\&A, 359, 669

Asplund, M., Nordlund, Å., Trampedach, R., Allende Prieto, C., \& Stein, R. F. 2000b, A\&A, 359, 729
Asplund, M., Grevesse, N. \& Sauval, A. J. 2005, in Cosmic Abundances as Records of Stellar Evolution and Nucleosynthesis, ed. T. G. Barnes, III \& F. N. Bash, ASP Conf. Ser., 336, 25

Barklem, P. S., Piskunov, N., \& O’Mara, B. J. 2000, A\&AS, 142, 467

Blackwell, D. E., Ibbetson, P. A., Petford, A. D., \& Willis, R. B. 1976, MNRAS, 177,219

Borrero, J. M. 2008, ApJ, 673, 470

Bray, R. J., Loughhead, R. E., \& Durrant, C. J. 1984, The solar granulation, 2nd edn. (Cambridge and New York: Cambridge University Press)

Chiavassa, A. 2008, in ed. S. Wolf, F. Allard, \& P. Stee, EAS Pub. Ser., 28, 31

Collet, R., Asplund, M., \& Trampedach, R. 2006, ApJ, 644, L121

Collet, R., Asplund, M., \& Trampedach, R. 2007, A\&A, 469, 687

Dravins, D. 1987a, A\&A, 172, 211

Dravins, D. 1987b, A\&A, 172, 200

Dravins, D. 2008, A\&A, 492, 199

Dravins, D., Lindegren, L., \& Nordlund, Å. 1981, A\&A, 96, 345

Freytag, B., Ludwig, H.-G., \& Steffen, M. 1996, A\&A, 313, 497

Gilliland, R. L., \& Dupree, A. K. 1996, ApJ, 463, L29

Gray, D. F. 1982, ApJ, 255, 200

Gray, D. F. 1984, ApJ, 277, 640

Gray, D. F. 1986, PASP, 98, 319

Gray, D. F. 2005, PASP, 117, 711

Gray, D. F., \& Nagel, T. 1989, ApJ, 341, 421

Gray, D. F., \& Toner, C. G. 1985, PASP, 97, 543

Gray, D. F., Carney, B. W., \& Yong, D. 2008, AJ, 135, 2033

Grevesse, N., \& Sauval, A. J. 1998, Space Sci. Rev., 85, 161

Gustafsson, B., Bell, R. A., Eriksson, K., \& Nordlund, А. 1975, A\&A, 42, 407

Gustafsson, B., Edvardsson, B., Eriksson, K., et al. 2008, A\&A, 486, 951

Hubeny, I. 1988, Computer Phys. Comm., 52, 103

Hubeny, I., \& Lanz, T. 1995, ApJ, 439, 875

Janssen, K., \& Cauzzi, G. 2006, A\&A, 450, 365

Koesterke, L., Allende Prieto, C., \& Lambert, D. L. 2008, ApJ, 680, 764

Kurucz, R. L. 1979, ApJS, 40, 1

Kurucz, R. L. 1993a, ATLAS9 Stellar Atmosphere Programs and $2 \mathrm{~km} \mathrm{~s}^{-1}$ grid. Kurucz CD-ROM (Cambridge, Mass.: Smithsonian Astrophysical Observatory), 13

Kurucz, R. L. 1993b, SYNTHE Spectrum Synthesis Programs and Line Data. Kurucz CD-ROM (Cambridge, Mass.: Smithsonian Astrophysical Observatory), 18

Ludwig, H.-G., Freytag, B., \& Steffen, M. 1999, A\&A, 346, 111

Mihalas, D., Dappen, W., \& Hummer, D. G. 1988, ApJ, 331, 815

Muller, R. 1999, in Motions in the Solar Atmosphere, ed. A. Hanslmeier, \& M. Messerotti, ASSL, 239, 35

Nordlund, Å. \& Dravins, D. 1990, A\&A, 228, 155

Nordlund, A., Stein, R. F., \& Asplund, M. 2008, Living Reviews in Solar Physics, in press

Ramírez, I., Allende Prieto, C., Koesterke, L., Lambert, D. L., \& Asplund, M. 2009, in preparation (Paper III)

Ramírez, I., Allende Prieto, C., \& Lambert, D. L. 2007, A\&A, 465, 271

Ramírez, I., Allende Prieto, C., \& Lambert, D. L. 2008, A\&A, 492, 841, (Paper I)

Robinson, F. J., Demarque, P., Li, L. H., et al. 2003, MNRAS, 340, 923

Stein, R. F., \& Nordlund, A. 1998, ApJ, 499, 914

Trampedach, R. 2007, AIP Conf. Ser., 948, 141

Vögler, A. 2004, A\&A, 421, 755 